\title{
THE FOUR-TERMINAL CONDUCTOR AND THE THOMSON BRIDGE
}

\author{
By Frank Wenner
}

\section{CONTENTS}

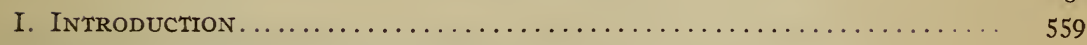

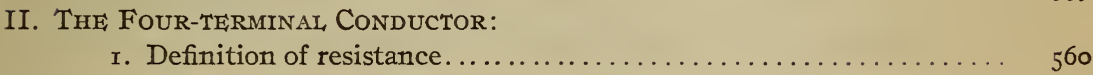

2. Definition of inductance.......................... ${ }_{562}^{62}$

3. The reciprocal theorem............................ ${ }_{563}^{6}$

4. Relation between resistances........................ ${ }_{569}$

5. The design of standards of resistance.................. 57 I

6. Standards for use with alternating current.............. 577

III. THE THOMSON BRIDGE:

I. Theory with linear conductors...................... ${ }_{580}$

2. The correction terms...........................

3. Adjustments making correction terms small............... $\quad 584$

4. An illustrative problem........................... $\quad 5^{86}$

5. Sensitivity with D'Arsonval galvanometer................ $\quad 5^{89}$

6 . The multiple bridge ............................ 595

7. Theory with nonlinear conductors. .................... $\quad 596$

8. Theory using alternating current $\ldots \ldots \ldots \ldots \ldots \ldots \ldots \ldots \ldots \ldots \ldots$

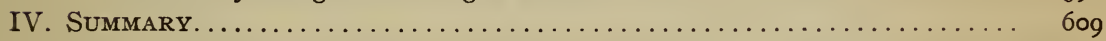

\section{INTRODUCTION}

The increased demand for the accurate measurement of power has necessitated the construction of low-resistance standards capable of carrying large currents. In such standards the surfaces of the contacts through which the current enters and leaves must be large, otherwise the heating will be excessive. Using different current leads or making the connections under different conditions necessarily introduces some variations in the current distribution in the terminals. On account of the relative proportions of the standard these changes are often not limited to the 
terminals, but extend to parts of the conductor between the potential terminals. As a result the resistance often depends, to some extent, on the manner in which the current leads are attached. Yet the standard, to be reliable, must have a definite value which must be determined in terms of the values of other standards.

Where the resistances are to be used in alternating-current measurements, it is also necessary to know the inductance, or to know that at the frequency used the phase angle between the current and the drop in potential is so small that it may be considered zero.

Various methods have been proposed or used in the comparison of the resistances of four-terminal conductors. One of these, known as the Thomson bridge method, has been in use a half century. In many cases where it is being used, moreover, sufficient precautions are not taken to get the accuracy easily attainable. However, this method has not come into use in proportion to its merits.

In considering the four-terminal conductor it is the purpose of this paper to point out the conditions which must necessarily be fulfilled in order that the resistance be definite or both the resistance and inductance be definite and to discuss some of the points to be observed in the design of low-resistance standards which are to carry large currents, especially if the current is alternating. In connection with the Thomson bridge we shall consider first the theory where the four-terminal conductors are linear, then discuss some of the ways in which the measurements have been made in the past, describe the way in which they are carried out at the Bureau of Standards, and show that the method being used is equally applicable where the four-terminal conductors are not linear, and finally consider adjustments which should be made when using alternating current.

\section{THE FOUR-TERIMINAL CONDUCTOR}

\section{DEFINITION OF RESISTANCE}

The generalized four-terminal conductor is a mass of conducting material of any size or shape and has four limited portions of the 
surface arbitrarily selected and adapted for making electrical connection to other conductors. In what follows we shall refer to these four limited portions of the surface (see Fig. I) as terminals I, 2, 3, and 4; that is, we shall limit the meaning of the word terminal to that part of the surfaces of the conductors adapted for making electrical connections. If a current enters and leaves the conductor through any two of the terminals, there is in general a difference in potential between the other two. The ratio of this difference in potential to the current may be considered one of the resistances of the conductor.

We shall define the ratio of the drop in potential from $I$ to 2 to the current entering at 3 and leaving at 4 as the resistance I-2/3-4, likewise the ratio of the drop in potential from 3 to $x$ to the current entering at 4 and leaving at 2 as the resistance $3-\mathrm{I} / 4-2$, etc.; in all, 24 resistances. We also have the resistances $3-2 / 3-4,2-3 / 2-3, \mathrm{I}-2 / 4-2$, etc. In these cases, however, only two or three of the terminals are used, so these resistances will not be considered in this paper, except in so far as may be necessary for a complete understanding of the case

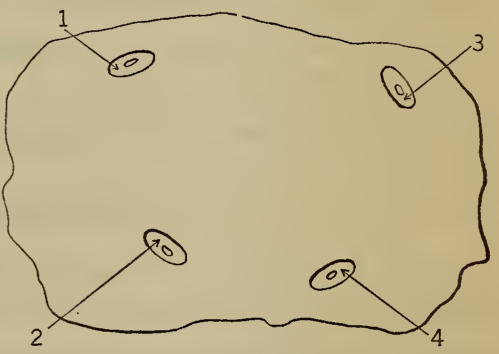

Fig. 1 where the four terminals are used. These various resistances and the relations between them have been considered fully in a recent paper by Searle. ${ }^{1}$

With the current entering at I and leaving at 4, 2 may be either at a higher or at a lower potential than 3 . The resistance $2-3 / \mathrm{I}-4$ may therefor be either positive or negative. As the notion of sign is foreign to our usual conception of resistance, we may, if we wish, consider it as associated with the drop in potential and with the direction of the current.

It will readily be seen that certain of the resistances are equal or have the same magnitude. For example

$$
\begin{aligned}
& 3-\mathrm{I} / 4-2=-\mathrm{I}-3 / 4-2=\mathrm{I}-3 / 2-4=-3-\mathrm{I} / 2-4 \\
& \mathrm{I}-2 / 3-4=-2-\mathrm{I} / 3-4=2-\mathrm{I} / 4-3=-\mathrm{I}-2 / 4-3, \text { etc. }
\end{aligned}
$$


Therefore, instead of 24 different values there can not be more than 6. We shall see later that there are other relations between these six values, so that only two are really independent.

Obviously, if any one of these resistances is to have a definite value, the ratio of the drop in potential between two of the terminals to the current entering and leaving by the other two must be independent of the part of each of the four terminals used in making electrical connections to the current and potential leads; that is, all parts of the terminals to which the potential leads are connected must be at the same potential and this potential must not be affected by the current density in the other two terminals so long as the total current is constant. From relations which will be shown later it follows that the only condition necessary for all the resistances to have a definite value is that there be no difference in potential between the parts of any one of the terminals, no matter what current may be entering and leaving the conductor through any two of the remaining terminals.

\section{DEFINITION OF INDUCTANCE}

If the current is changing, there is, in general, an additional difference in potential between the potential terminals. The ratio of this additional difference in potential to the rate of change of the current is the inductance. However, in using the four-terminal conductor, leads must be connected to each of the four terminals and there is no way by which to distinguish between the emf developed (by mutual inductance) in the two potential leads which do not carry a current and the emf which is developed between the terminals to which they are connected. There is, therefore, no reason for separating the emf developed between the terminals from the total emf in the circuit composed of the two leads and the part of the conductor between the two terminals to which they are connected. We may, therefore, define the inductance of a four-terminal conductor as the ratio of the emf induced in a circuit composed of the conductor and one pair of leads to the rate of change of the current in a circuit composed of the conductor and the other pair of leads. It is to be understood 
that each of these circuits is closed or practically closed. Obviously, the number of inductances is the same as the number of resistances and can be designated in the same way.

If any one of these inductances is to have a definite value, the terminals must either be brought out in such a way that the mutual inductance between the two pairs of leads may be made negligible or the parts of the leads which contribute to the mutual inductance between the two circuits must always be arranged in the same definite relative positions.

\section{THE RECIPROCAL THEOREM}

Before proceeding to the consideration of the less obvious relations between the resistances and inductances obtained by using the four terminals in different combinations, it will be necessary to call attention to a general theorem. This theorem may be stated as follows: In any conductor or system of conductors having four terminals $\mathrm{I}, 2,3$, and 4 selected in any way, the drop in potential from $\mathrm{I}$ to 2 caused by a current entering at 3 and leaving at 4 is equal to the drop in potential from 3 to 4 caused by an equal current entering at $I$ and leaving at 2 .

For a network of linear conductors this theorem was given by Kirchhoff ${ }^{2}$ in the same paper in which he gave the laws in regard to the sum of the currents to a point and the emf and current in a colsed circuit.

For an isotropic and homogeneous nonlinear conductor this theorem was developed theoretically by Helmholtz. ${ }^{3} \mathrm{He}$ also considered the case of a conductor having two parts of different conductivities and tested the relation experimentally on a carbon cylinder 3.5 inches long and 2 inches in diameter. In this work electrical connections were made by means of four small quantities of mercury held in place by paper rings, and the currents compared by reading the deflections of a galvanometer. Rosen ${ }^{4}$

\footnotetext{
2 Pogg. Ann., 72; 1847. Kirchhoff, Ges. Abh., p. 32. See also Maxwell Ellec. and Mag., 1, p. 371, and Gray Abs. Meas., 1, p. 160 .

3 Pogg. Ann., 89, p. 2 r1; r853. Helmholtz, Wiss. Abh., 1, p. 496。

4 Öfvers. af k. Vetensk. Akadem. Vorhandl., p. 197; r887.
} 
extended the proof of this theorem so as to include the case of nonhomogeneous and nonisotropic conductors. In the paper by Searle, referred to above, two proofs of the theorem are given, one by Heaviside and one by Bromwich, both of which are somewhat similar to that given by Rosen.

In order to extend the theorem to the case where the current is alternating it will be necessary to consider the whole matter further. For the simple case (Case I, below) where the conductor is isotropic and the current constant the proof which will be given, though developed independently, may be considered practically the same as that given by Rosen, Heaviside, and Bromwich. In considering this matter a vector notation will be used in which all vectors are designated by bold-faced type and scalar products indicated by a dot $(\cdot)$ between the vectors.

According to the theorem of Gauss, sometimes referred as the divergence theorem ${ }^{5}$

$$
\int \boldsymbol{F}_{n} \delta s=\int \nabla \boldsymbol{F} \delta v
$$

where $\boldsymbol{F}$ is a finite, single valued, and continuous vector function of space, $\delta v$ an element of volume, $\delta s$ an element of surface and $\boldsymbol{F}_{n}$ is the component of $\boldsymbol{F}$ normal to the surface. Here the surface integral is to be extended over a closed surface and the volume integral extended through the volume inclosed by the surface.

Consider a four-terminal conductor so designed as to have definite resistances ${ }^{6}$ and let the surfaces over which we are to integrate (see Fig. 2) include all of the conductor but coincide with the terminals $I, 2,3$, and 4 .

Let $\psi$ be the potential at any point above that at 3 caused by a current $I$ entering at $I$ and leaving at 4 ,

$\phi$ be the potential at any point above that at 4 caused by a current $C$ entering at 2 and leaving at 3 ,

$i$ be the density at any point of the current $I$, $c$ be the density at any point of the current $C$.

For the derivation of this equation and equations similar to those which follow, see Abraham und Föppl Theorie der Electrizitite, 1, p. 54 .

6 For explanation of definite resistance, see p. $5^{62}$. 
Now if we substitute $\phi \boldsymbol{i}$ for $\boldsymbol{F}^{7}$ we have

$$
\int(\phi i)_{n} \delta s=\int \nabla(\varphi i) \delta v
$$

and if we substitute $\psi c$ for $\boldsymbol{F}$ we have

$$
\int(\psi c)_{n} \delta s=\int \nabla(\psi c) \delta v
$$

Carrying out the indicated differentiation of the right-hand members of these equations and subtracting gives

$$
\int(\psi c)_{n} \delta s-\int(\phi i)_{n} \delta s=\int(\psi \nabla c-\phi \nabla i) \delta v+\int(c \nabla \psi-i \nabla \phi) \delta v .
$$

In regard to the surface integrals, the current density $c$ is zero everywhere except on 2 and 3 while $\psi$ is zero on 3 and constant on 2 . The integration of $\boldsymbol{c}_{n}$ over the surface of the terminal 2 gives the total current $C$ or the value of the first integral of equation (5) is $-\psi_{2} C$. Likewise the value of the second integral is $\phi_{1} I$ or

$$
\int(\psi c)_{n} \delta s-\int(\phi i)_{n} \delta s=\phi_{1} I-\psi_{2} C \text {. }
$$

The value of the right-hand member of equation (5) will depend

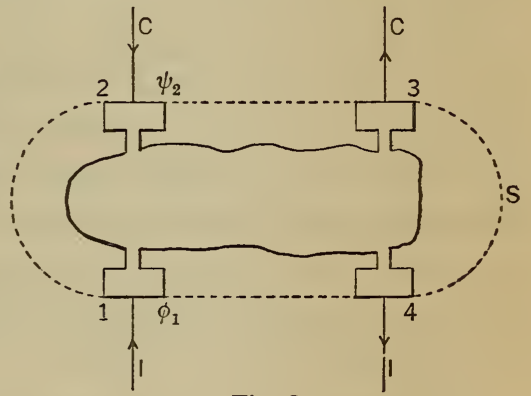

Fig. 2 upon a number of conditions or set of conditions, of which we will consider four special cases. In each case we shall consider that the resistance is definite, and that the conductor is free from internal sources of current.

Case I: Conductor isotropic and current constant.

If $\rho$ represents the volume resistivity,

$$
\begin{aligned}
& -\nabla \phi=c \rho \text { and }-\nabla \psi=i \rho \\
& \therefore c \nabla \psi-i \nabla \phi=0
\end{aligned}
$$

for every point in the conductor. The conductor being free from sources of current, $\nabla \boldsymbol{i}$ and $\boldsymbol{\nabla} \boldsymbol{c}$ are equal to zero for every point

${ }^{7}$ For the conditions which the functions $F, \psi$, and $\phi$ must fulfill, see Peirce, Newtonian Potential Function, p. 93 . 
within the conductor. Therefore the entire second member of the equation is zero and we have

$$
\varphi_{1} I-\psi_{2} C=0
$$

or the resistance $I-4 / 2-3$ equals the resistance $2-3 / \mathrm{I}-4$.

Case II: Conductor nonisotropic or crystalline, current constant. The first volume integral is zero for the same reason as in Case I. In each individual crystal or nonisotropic element of volume there is, in general, an angle between the potential gradient and the current density. There are, however, in most if not all such cases three directions in which if a potential gradient is impressed the current will be in the same direction. These directions may be considered the electrical axes of the element of the conductor and the current densities $\boldsymbol{i}$ and $\boldsymbol{c}$ may be resolved into components $\boldsymbol{u} i_{u}, \boldsymbol{v} i_{v}, \boldsymbol{w} i_{w}, \boldsymbol{u} c_{u}, \boldsymbol{v} c_{v}$, and $\boldsymbol{w} c_{w}$ parallel to these axes. Here $\boldsymbol{u}, \boldsymbol{v}$ and $\boldsymbol{v}$ are unit vectors parallel to the axes and $i_{u}, i_{v}, i_{w}, c_{u}, c_{v}, c_{w}$, the magnitudes of the componeints of the currents. If, then, we let $\rho_{u}, \rho_{v}$, and $\rho_{w}$ be the resistivity in directions parallel to the axes, we have

$$
\begin{gathered}
c \nabla \psi-i \nabla \phi=u \cdot v\left(i_{u} c_{v}-i_{v} c_{u}\right)\left(\rho_{v}-\rho_{u}\right) \\
+\boldsymbol{u} \cdot \boldsymbol{v}\left(i_{u} c_{w}-i_{w} c_{v}\right)\left(\rho_{w}-\rho_{u}\right)+\boldsymbol{v} \cdot \boldsymbol{v}\left(i_{v} c_{w}-i_{w} c_{v}\right)\left(\rho_{w}-\rho_{v}\right) .
\end{gathered}
$$

If either (I) the axes are mutually perpendicular, (2) the resistivity is the same for current in the direction of all of the three axes, or (3) the resistivity is the same for current in the direction of two axes which are themselves perpendicular to the third axis, then

$$
c \Delta \psi-i \Delta \varphi=0
$$

for every point, the same as in an isotropic conductor, and the resistance $\mathrm{I}-4 / 2-3$ is equal to the resistance $2-3 / \mathrm{I}-4$.

Here it is shown that certain conditions are necessary in order that the theorem may be true. It is known, however, from experiment that most, if not all, conductors fullfil at least one of these conditions. In the papers referred to above it is pointed out that in order for the theorem to be true it is necessary that if a current density $i_{x}$ in the direction of the $x$ axis requires a com- 
ponent of the potential gradient $e_{y}$ along the $y$ axis then a current density $i_{y}$ along the $y$ axis must require a component of the potential gradient $e_{x}$ along the $x$ axis, and $e_{x} / i_{y}$ must be equal to $e_{y} / i_{x}$. Also similar relations must exist between the other components. This relation between the current density and the components of the potential gradient in the element of volume is almost identically the relation with which we are concerned for the four-terminal conductor as a whole.

Case III: Conductor isotropic, current changing.

Here

$$
c \Delta \psi=-c \cdot i \rho-c \cdot m_{i} \frac{\delta I}{\delta t}
$$

and

$$
i \Delta \varphi=-i \cdot c \rho-i \cdot m_{c} \frac{\delta C}{\delta t}
$$

where $\boldsymbol{m}_{i}$ is the mutual inductance of the total circuit in which the current $I$ flows upon a unit length of the conductor taken in a direction parallel to the induced emf. As the direction of the induced emf has no relation to the direction of either of the current densities $\boldsymbol{i}$ or $\boldsymbol{c}, \boldsymbol{m}_{i}$ must be considered as a vector quantity. Likewise the $m_{c}$ is the mutual inductance of the total circuit in which the current $C$ flows upon a unit length of the conductor taken in a direction parallel to the emf induced by changes in the current $C$. Therefore

$$
\int(\boldsymbol{c} \Delta \psi-i \Delta \phi) \delta v=\frac{\delta C}{\delta t} \int i \cdot m_{c} \delta v-\frac{\delta I}{\delta t} \int \mathfrak{c} \cdot m_{i} \delta v .
$$

For the first integral of the second member we may take as the element of volume the part of the conductor included between two equipotential surfaces in one of the tubes of current constituting a part of the current $I$. If $\delta i^{\prime}$ is the current in the tube, $\delta a$ the area of the tube at the point in question, and $\delta l$ the distance between equipotential surfaces then

$$
i \delta a=\delta \boldsymbol{i}^{\prime} \quad \text { also } \delta v=\delta a \delta l
$$

or

$$
\frac{\delta C}{\delta t} \int i \cdot \boldsymbol{m}_{c} \delta v=\frac{\delta C}{\delta t} \iint \boldsymbol{m}_{c} \cdot \delta \boldsymbol{i}^{\prime} \delta l=I M_{c} \frac{\delta C}{\delta t}
$$


likewise

$$
\frac{\delta I}{\delta t} \int \boldsymbol{c} \cdot \boldsymbol{m}_{i} \delta v=C M_{i} \frac{\delta I}{\delta t}
$$

where $M_{i}$ is the mutual inductance of the total circuit in which the current $I$ flows upon the part of the circuit between terminals in which the current $C$ flows. Also $M_{c}$ is the mutual inductance of the total circuit in which the current $C$ flows upon the part between the terminals of the circuit in which the current $I$ flows.

From this it follows that

$$
\varphi_{1} I-\psi_{2} C=I M_{c} \frac{\delta C}{\delta t}-C M_{i} \frac{\delta I}{\delta t}
$$

If the connectors and leads are so arranged that the leads contribute nothing to the mutual inductance $M$ between the two circuits in which the current $I$ and $C$ flow, and if the current distribution is the same with changing as with constant current, then $M_{c}=M_{i}=$ $M$. Or, if the parts of the leads near the conductor contribute to the mutual inductance between the two circuits, the inclosing surface may be enlarged so as to include these parts of the leads, in which case $M_{c}=M_{i}=M$. If, further, both $I$ and $C$ are alternating currents of the same frequency and wave form, then $M_{c}=M_{i}=M$ even though the current distribution is affected by the frequency. Therefore,

since.

$$
I=I^{\prime} \epsilon^{p t} \text { and } \frac{\delta I}{\delta t}=\iota p I^{\prime} \epsilon^{\prime p t}
$$

and since

$$
C=C^{\prime} \epsilon^{\phi p t} \text { and } \frac{\delta C}{\delta t}=\iota p C^{\prime} \epsilon^{\phi p t}
$$

where $I^{\prime}$ and $C^{\prime}$ are proportional to the maximum values of the currents, $p=2 \pi$ times the frequency, $\iota=\sqrt{-1}$ and $\epsilon$ is the base of natural logarithm; we have

$$
M\left(I \frac{\delta C}{\delta t}-C \frac{\delta I}{\delta t}\right)=\mathbf{0}
$$

and the impedence $I-4 / 2-3$ is equal to the impedence $2-3 / \mathrm{I}-4$. Therefore, to alternating currents of the same frequency, the resistances $I-4 / 2-3$ and $2-3 / I-4$ are equal. 
Case IV: Conductor nonisotropic, current changing.

Since we have here only a combination of Cases II and III, it follows that, with the limitations of both of these cases, the theorem is applicable here.

In showing that the theorem is applicable to such conductors and under such conditions as we may find in resistance measurements it has. been necessary to assume a conductor free from sources of current (such as thermoelectromotive forces, the Hall, and similar effects) that there are at least three axes in each element of volume along which the current flows parallel to the potential gradient, and either that these three axes are mutually perpendicular, or the conductivities to current along each of the three axes are equal, or the conductivities to current along each of two axes are equal and these two axes are both perpendicular to the third. In order for the theorem to be applicable in case the current is alternating it has been shown that the total mutual inductance between the two circuits must be taken into consideration.

The effect of electrostatic capacity has not been considered, since, in the case of resistance standards designed to carry large currents, it is known to have no appreciable effect. Furthermore, in the case of resistance coils the effect of capacity is known to be the opposite to the effect of a self-inductance.

We shall make use of this theorem not only in the consideration of the relations between the different resistances and inductances of the four-terminal conductor but more particularly in showing the relations between the resistances and inductances of the Thomson bridge.

\section{RELATION BETWEEN RESISTANCES}

Making use of the reciprocal theorem we have the following relations between the six resistances considered above

$2-3 / \mathrm{I}-4=\mathrm{I}-4 / 2-3, \mathrm{I}-2 / 4-3=4-3 / \mathrm{I}-2$, and $\mathrm{I}-3 / 2-4=2-4 / \mathrm{I}-3$

This shows that in case no two of the four leads are connected to the same terminal and considering only positive values there are three and only three different resistances.

$$
56109^{\circ}-\mathrm{r} 2-9
$$


In order to show the further relation between the three values we shall consider the ratio of the difference in potential between the different terminals to the current. If, for example, the current enters at $I$ and leaves at 4 , the differences in potential between I and 4, 2 and 4, 3 and $4, I$ and 2, I and 3, and 2 and 3 , divided by the current are the resistances I-4/I-4, 2-4/I-4, 3-4/I-4, I-2/I-4, I- $3 / \mathrm{I}-4$, and $2-3 / \mathrm{I}-4$.

As the difference in potential between 2 and 3 is the difference in potential between 2 and 4 minus the difference in potential between 3 and 4 , we have the following relation between the resistances:

Likewise

$$
2-3 / \mathrm{I}-4=2-4 / \mathrm{I}-4-3-4 / \mathrm{I}-4
$$

and

$$
\begin{aligned}
& \mathrm{I}-3 / 2-4=\mathrm{I}-4 / 2-4-3-4 / 2-4 \\
& 2-\mathrm{I} / 3-4=2-4 / 3-4-\mathrm{I}-4 / 3-4
\end{aligned}
$$

Adding (I9) and (20) gives

$$
\mathrm{I}-3 / 2-4+2-\mathrm{I} / 3-4=\mathrm{I}-4 / 2-4-\mathrm{I}-4 / 3-4=2-4 / \mathrm{I}-4-3-4 / \mathrm{I}-4 \text {. }
$$

since $3-4 / 2-4=2-4 / 3-4, \quad I-4 / 2-4=2-4 / I-4$, and $I-4 / 3-4=3-4 / I-4$. Therefore, from (I8) and (2I) it follows that ${ }^{8}$

$$
2-3 / \mathrm{I}-4=\mathrm{I}-3 / 2-4+2-\mathrm{I} / 3-4
$$

Four terminal conductors which are to be used as resistance standards, ammeter shunts, etc., usually have two of the terminals arranged for connection to the current leads and two for connection to the potential leads. For convenience we may designate the potential terminals as 2 and 3 , and the current terminals as I and 4 , 2 being the potential terminal at the higher potential when the current enters at $\mathrm{r}$. The resistance with which we are most concerned, then, is $2-3 / \mathrm{I}-4$ (or $\mathrm{I}-4 / 2-3$ ), and in what follows we shall refer to it as the direct resistance or simply as the resistance. The resistance $2-1 / 3-4$ or $3-4 / 2-I$ we shall refer to as the cross resistance, and the resistance $2-4 / \mathrm{I}-3$ or $\mathrm{I}-3 / 2-4$ we shall refer to as the diagonal resistance. From equation (22) it follows that the resistance is equal to the cross resistance plus the diagonal resist- 
ance. Where the cross resistance is negligibly small in comparison with the direct resistance the diagonal and direct resistance are equal. In this case, if the conductor is connected into any system of conductors, the same effects are obtained as would be obtained with a conductor having definite branch points joined by a singlewire conductor. Such a conductor we shall refer to as linear to distinguish it from the more general case.

In most well-designed resistance standards the ratio of the cross to the direct resistance is small. For the smaller-size standards of the Reichsanstalt type constructed by Otto Wolff and having values of $0.00 \mathrm{ohm}$ and above, this ratio is generally less than $10^{-6}$. Such standards may therefore be considered as linear. Even where the value is o.ooor ohm the ratio is only a few hundredths per cent.

In standards of low resistance, if the potential connectors are located at or near the side of the current connectors, the ratio is often of the order of I per cent and may readily be much larger.

While the inductance $2-3 / \mathrm{I}-4$ equals the inductance $\mathrm{I}-4 / 2-3$, etc., we can not have the same simple relations between the direct, cross, and diagonal inductances as between the corresponding resistances, since in changing from one to the other the relative position of the leads and consequently their mutual inductance must be changed.

\section{THE DESIGN OF STANDARDS OF RESISTANCE}

The main feature to be considered here is a construction such as will make the resistances definite without making the mass unnecessarily large. The condition necessary can only be realized approximately, yet we shall see that we can make the direct resistance at least as definite as it can be measured. A matter of secondary importance is to make the cross resistance small in comparison with the direct resistance.

With terminals of a given size, shape, and relative location, the condition for a definite resistance is more nearly fulfilled the higher the conductivity of the material used in making the connectors, and with infinite conductivity the resistance would all be definite. For other reasons copper is generally used for 
the connectors of the lower resistances. As little could be gained by the substituting of any other material for copper, even if the matter of cost were not to be considered, this suggestion can lead to few if any improvements in the design of such conductors.

In the more usual cases the condition is more nearly realized the smaller the terminals are made. With very small terminals the potential is necessarily the same all over each terminal when there is no current through it. Reducing the section of the connectors even for a very short length has the same effect as reducing the size of the terminals. The area of the potential terminals or section of the potential connectors may be made very small, since they are seldom required to carry much current. On the other hand, the area of the current terminals can not be made small nor the connectors of small section even for a short length on account of the large current which will sometimes be used. Nevertheless, many resistance standards, ammeter shunts, etc., could be materially improved by a judicious reduction of the section of the current connectors, even though doing so makes it necessary to dissipate a larger amount of power in the conductor. Ordinarily the potential connectors have a sufficiently small section so that little if anything would be gained by a further reduction of their section.

The effect of reducing the area of the terminals or the minimum section of the connectors is to make the current distribution throughout the major part of the conductor more nearly independent of the current distribution on the terminals, through which the current enters and leaves. Thus the same effect may be obtained by increasing the length ${ }^{9}$ of the connectors. But this not only increases the amount of power which must be dissipated in the conductor for a given current, but also increases the mass and cost, especially in the case of conductors intended to carry very large currents.

Experiments made by the author show that for a terminal of a definite size and shape the current distribution is more nearly independent of the part of the terminal used if the connector is

9 The effect of the relative dimensions of the connectors on the definiteness of the resistance is discussed fully by Searle: Electrician, 66, p. 1032, and 67, p. 12; 1911. 
made of materials having widely different conductivities, using the material of lower conductivity between portions of the material of higher conductivity. The material of the lower conductivity, then, has very much the same effect as would be obtained by making the connector longer and using the material of the higher conductivity throughout. While in many cases improvements might be made either by reducing the area of the terminals or the section near the middle of the connectors, by increasing the length of the connectors, or by making the connectors of materials of different conductivities, yet for conductors which are to carry large currents better results will be obtained by working along somewhat different lines.

A matter which has considerable effect upon the definiteness of the resistances is the relative locations of the different terminals. This will readily be understood by reference to Fig. 3 , which shows two heavy conductors used as terminal blocks connected by three nearly equal wire resistances. Near the junction

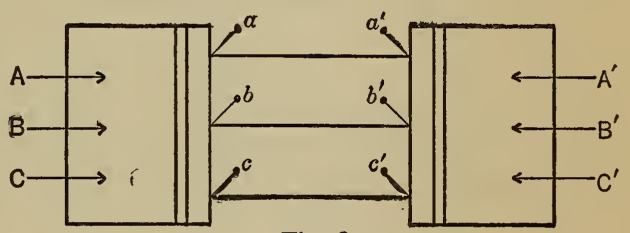

Fig. 3 of the wires and terminal blocks are brought out six terminals $a$, $b, c, a^{\prime}, b^{\prime}$, and $c^{\prime}$, the whole constituting a conductor with large current terminals and three pairs of potential terminals. The different potential connectors being of small section, the potential must necessarily be constant over the terminals when there is no current through them. Current entering and leaving through the current terminals may not divide equally between the three wire conductors, so the potential difference between $a$ and $a^{\prime}, b$ and $b^{\prime}$, and $c$ and $c^{\prime}$ is not necessarily the same. If $a$ and $a^{\prime}$ are used as the potential terminals and the major part of the current enters the conductor in the vicinity of $A$ and leaves in the vicinity of $A^{\prime}$, then more than one-third of the current will flow through the conductor (I) and consequently the resistance will be more than one-third the mean value. If, on the other hand, the current enters mainly in the vicinity of $C$ and leaves mainly in the vicinity of $C^{\prime}$, then the resistance will be less than one-third the mean value. 
Again, if $b$ and $b^{\prime}$ are used as potential terminals, the resistance is very nearly the same whether the current enters in the vicinity of $A$ or $C$ and leaves in the vicinity of $A^{\prime}$ or $C^{\prime}$. There is, therefore, a decided advantage in having the connectors to the potential terminal brought out symmetrically with respect to the parts of the current terminal.

It will be observed, however, that if the current enters and leaves in the vicinity of $B$ and $B^{\prime}$ the resistance will be higher than if it enters and leaves in the vicinity of $A$ or $C$ and $A^{\prime}$ or $C^{\prime}$. Obviously, if we can prevent the current from entering and leaving in the vicinity of $B$ and $B^{\prime}$, the resistance of the conductor will be more nearly definite. This may be accomplished by cutting away the part of the terminals including the area around $B$ and $B^{\prime}$. The same result may be accomplished without an appreciable reduction of the area of the terminals by cutting away a portion

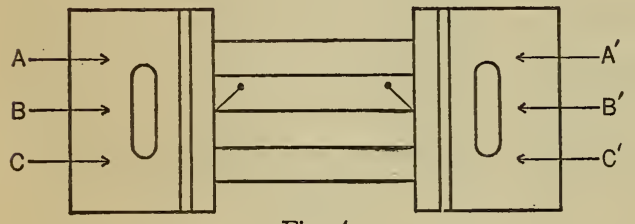

Fig. 4

of the connector, as shown in Fig. 4. In this case the current entering and leaving at $B$ and $B^{\prime}$ necessarily distributes itself much as it would with half entering at $A$ and leaving at $A^{\prime}$ and the other half entering at $C$ and leaving at $C^{\prime}$. That is, the proportional part of the current carried by the center conductor is about the same as it would be with the current entering at $A$ or $C$ and leaving at $B$ or $B^{\prime}$. With this arrangement the resistance is much more nearly definite than with the arrangement shown in Fig. 3, especially if $a$ and $a^{\prime}$ or $c$ and $c^{\prime}$ are to be used as potential terminals.

It will easily be seen that the addition of two nearly equal linear conductors symmetrically located, as shown in Fig. 4, does not make the resistance of the system any more indefinite. Likewise, if instead of any number of pairs of equal linear conductors symmetrically located we have a single uniformly conducting sheet, the resistance is slightly more definite.

Where the resistance material is in the form of sheets a number of which are set in parallel the parts of the current terminals 
should be arranged symmetrically with respect to the individual sheets and also in respect to the whole number of sheets. Also the potential connector should be brought out from a point about which the rest of the conductor, including the current terminals, is symmetrical in two planes at right angles to each other.

With potential terminals on the side the indefiniteness of the resistance of the conductors designed to carry large currents has been found to amount to as much as one-half per cent and might easily amount to more. In limiting the part of each current terminal to two not very small areas symmetrically located and using symmetrically located potential terminals the indefiniteness of the resistance of these same conductors became so small that it could scarcely be detected. The advantage of locating the terminals and connectors symmetrically is that the resistance is made much more definite without increasing either the mass or the resistance of the current connectors.

Another way of attacking the problem is the use of branched potential con-

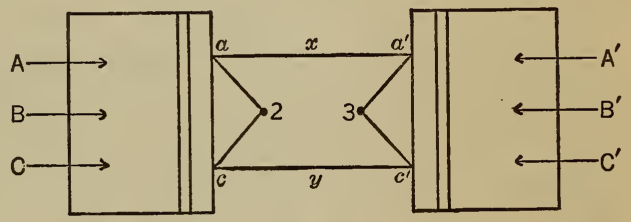

Fig. 5 nectors. The advantage of such an arrangement will readily be understood by reference to Fig. 5, which shows two heavy conductors connected by two wire conductors of practically equal resistance. The ends of the wire conductors are connected, as shown, by approximately equal resistances to the terminals 2 and 3 and the whole constitutes a four-terminal conductor. With this arrangement the difference in potential between the potential terminals 2 and 3 is very approximately the mean of the difference in potential between the points $a$ and $a^{\prime}$ and $c$ and $c^{\prime}$, regardless of the way the current divides between the two wires. Here, unless the resistance from $A$ to $B$ and from $A^{\prime}$ to $B^{\prime}$ is unnecessarily large, the part of the current carried by each wire changes by only a small amount (a few per cent at most) on using different parts of the current terminals. The indefiniteness of the resistance then must be exceedingly small, since even 
a large change in the current distribution produces only a small change in the resistance.

If the resistance from $a$ through 2 to $c$ and from $a^{\prime}$ through 3 to $c^{\prime}$ is large in comparison with the resistance of the wires $x$ and $y$, four more conductors of nearly equal resistances may be added without materially affecting the definiteness of the resistance, if two are placed near and symmetrically with respect to $x$ and the other two are placed near and symmetrically with respect to $y$. This is necessarily the case, since any change in the current distribution, such as the increase in the part carried by $x$, will be accompanied by proportional increase of current in the two conductors near $x$ and a decrease of the current in the two conductors near $y$ in such a way that the fractional part of the total current carried by $x$ as compared with that carried by $y$ will be just about the same as it was before the change.

For conductors of the usual type where either wires or sheets are used the number of branches which the potential terminals may have is not limited to two but may be almost any number desired. If the resistance material is in the form of a uniform sheet, there may be two or more branches from each potential terminal connected near where the sheet joins the terminal block and spaced uniformly along the junction. Where there are a number of sheets in parallel a very definite value for the direct resistance would be obtained by having two or more branches from each terminal to each sheet. This, however, would make the construction very complicated and is an arrangement which need not be considered, since the use of good conducting material for the connectors, a fairly long connector, a symmetrical arrangement of the terminals, and branched potential connectors each supplement the other in making the resistance more definite. If in the design all these ideas are kept in mind, the resistance of conductors, even if they are to carry excessively large currents, can be made sufficiently definite for the most precise measurements without making the current connectors excessively heavy nor their resistance of the order of that of the four-terminal conductor. This can be accomplished, too, without carrying any one of these ideas to an extreme. 


\section{STANDARDS FOR USE WITH ALTERNATING CURRENT}

If the conductor is to be used as a standard of resistance in alternating current measurements, the more important requirements in addition to those for use in direct-current measurements are that the resistance shall be the same or nearly the same for alternating as for direct current and that direct inductance be small and definite. If the standard is to carry a large current, these additional requirements make the design a much larger problem, since the usual arrangement of a number of sheets in parallel can not be used and without such an arrangement it is difficult to get a sufficient surface through which to dissipate the heat developed in the resistance by the current. The first requirement is more nearly fulfilled if the material is made in the form of a tube ${ }^{10}$ having a thin wall and a considerable diameter. In this case, then, if the frequency is low, the current distribution and consequently the resistance will be nearly the same with alternating as with direct current. Further, if the current leads lie in the axis of the tube, the increase in resistance if sufficient to be considered, may be calculated to a fair accuracy. ${ }^{11}$ To get the necessary surface to keep the rise in temperature from becoming excessive requires a tube of large diameter and of considerable length. In general, a change in current distribution results in a change in the inductance. However, symmetrical location of the terminals or connectors and the use of branched potential connectors each have about the same effect in making the inductance definite as they have in making the resistance definite. The inductance may be made very small by making the potential connectors of considerable length and so locating ${ }^{12}$ them with respect to the circuit in which the current flows that the flux cutting across them, for any change in the current, is the same or nearly the same as that cutting across the resistance material between the points to which they are joined. If, then, the potential connectors extend some little distance from the main part of the

10 Patterson and Rayner: Collected Researches Nat. Phys. Lab., 6, p. 90; I9I0.

11 Russell: Phil. Mag., 17, p. 524; 1909.

12 Orlich: Zs. Instk., 29, p. 148; 1909. Campbell: Electrician, 61, p. I000; 1909. 
conductor and the terminals are close together, the potential leads can easily be arranged so that they contribute nothing to the mutual inductance between the current and potential circuits. In this case the inductance will be very small and definite and is as likely to have a negative as a positive sign.

The way in which these ideas may be embodied in the construction of resistance standards is illustrated by Fig. 6, which shows the

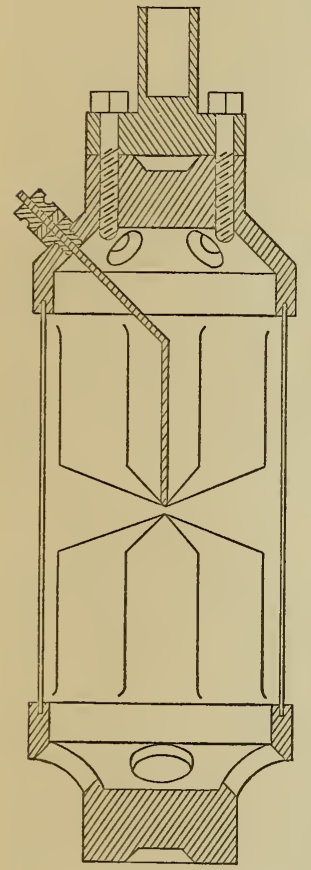

Fig. 6 design of a resistance standard for use with alternating current. The resistance is to be o.oor ohm and the current capacity, with a forced circulation of oil to assist in dissipating the heat developed, is to be 1000 amperes. The main part of the conductor is to be of manganin in the form of a tube $5 \mathrm{~cm}$ in diameter, about 25 $\mathrm{cm}$ long, and $.25 \mathrm{~mm}$ thick. The current connectors and the lugs for the current leads are to be of the best grade of cast copper. The potential connectors are each to have six or eight branches, and each branch is to have a resistance of $0.02 \mathrm{ohm}$. The branches of each potential connector are to be attached to the tube at points uniformly spaced along the junction between the connector and the tube. The potential connectors are to extend from the inside of the tube through an insulating bushing to the potential terminals, only one of which is shown in the figure. The other potential terminal and that part of the connectors on the outside of the tube are to be placed near the one shown, so that the flux linking this part of the potential circuit will be very small. This arrangement should make the inductance entirely negligible for measurements. with low-frequency alternating currents. The large amount of heat developed is to be dissipated by a vigorous circulation of oil over both the inside and outside surface of the tube. The circulation through the inside is made possible by having four large holes through both of the current connections. 
The oil bath, not shown in the figure, is to be circular and of slightly larger diameter than the standard. If it is desired to cool the oil, a coil of copper tubing may be placed inside the bath and water circulated through it. The bottom of the oil tank is to be made of cast copper, which can now be obtained very pure, and amalgamated on a part of the upper surface. Also the lower current terminal is to be amalgamated and electrical connection between the two made by means of mercury. The lower current lead is attached to the upper side of the tank and extends downward along the axis of the tube. The lower current connector is to be drilled and tapped like the upper one, so that for smaller currents the standard may be used without the oil bath.

This design was developed mainly with the idea of getting a standard suitable for use with the Thomson bridge in the determination of the inductance and resistance of other standards to be used in alternating-current measurements and of such form that both its inductance and change in resistance with frequency can be calculated. It is not presumed that such a standard will be found satisfactory for general use, mainly on account of the strong magnetic field produced even at a considerable distance from the axis of symmetry.

In considering the design of conductors to be used as standards of resistance we have given but little consideration to the cross resistance and none to the cross inductance. However, most of the suggestions made above for increasing the definiteness of the resistance also increase the definiteness of the cross resistance and some of them tend to make it smaller.

Bringing the petential connectors out in such a way so as to make the direct inductance very small results in making the cross inductance of considerable magnitude. But we are very much concerned in keeping the one small and care very little about the value of the other. In some of the measurements, in which a part of the current enters or leaves through the potential terminals, the cross inductance and the cross resistance have an effect, which, if not taken into account, may lead to errors. This matter will be considered further in connection with the theory of the Thomson bridge. In most measurements, however, neither the cross induc- 
tance nor the cross resistance can have any effect upon the results obtained.

\section{THE THOMSON BRIDGE}

\section{THEORY WITH LINEAR CONDUCTORS}

In I 862 Sir William Thomson described a method for the comparison of low resistances and pointed out many of its advantages. ${ }^{13}$ The arrangement of conductors which he used has since come to be known as the Thomson bridge, though it is often referred to as the Kelvin double bridge. The method has been modified from time

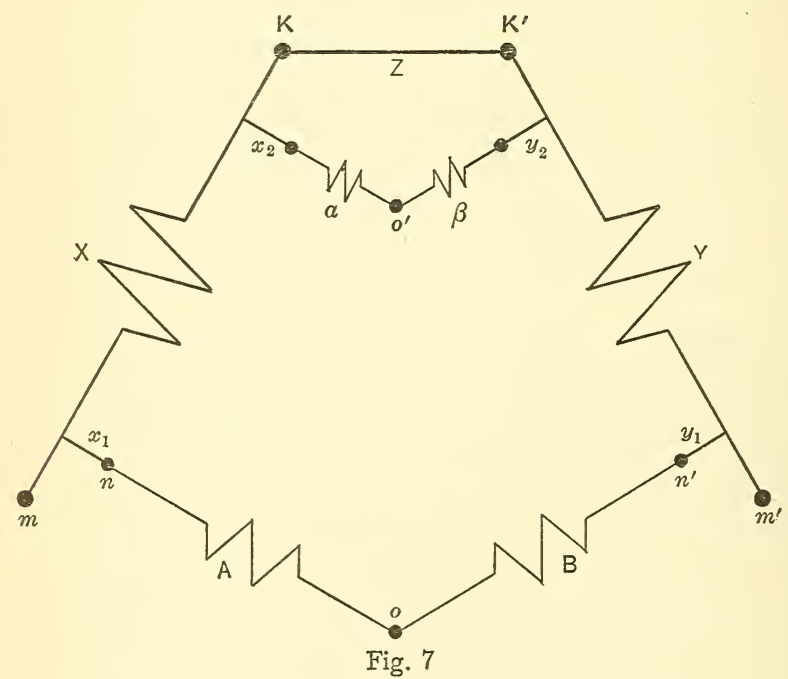

to time to adapt it to different classes of work, to get a higher precision, or to reduce the number of separate measurements necessary for determining the relation between the resistance and the standard with which it is compared.

The arrangement of conductors for a Thomson bridge is shown in Fig. 7. Here the low resistances under comparison are designated by $X$ and $Y$, the resistances for the main ratio by $A$ and $B$, and the auxiliary ratio by $\alpha$ and $\beta$. Further, the connecting resistances from the points where the current and potential terminals divide to the terminals of the ratio sets are designated by $x_{1}, x_{2}, y_{1}$, and $y_{2}$. These letters are used both to designate the conductors and to 
represent the values of these resistances. $C$ represents the value of the resistance between $X$ and $Y$ from branch point to branch point, while $Z$ designates the low resistance in parallel with the auxiliary ratio. If, then, the conductors are all linear and the bridge is balanced, we have the following relation between the resistances:

$$
X=Y^{A+x_{1}}+\frac{A+x_{1}}{B+y_{1}} C \frac{B+y_{2}}{\alpha+y_{1}}-C \frac{\alpha+x_{2}}{\alpha+\beta+x_{2}+y_{2}} .
$$

This equation can easily be established from relations which exist in the simple bridge if we observe that the resistance $C$ is divided in two parts having the same ratio as $\alpha+x_{2}$ to $\beta+y_{2}$ and that the first is in the same arm as $X$ and the second in the same arm as $Y$. Before considering the matter further we will put this equation in a more convenient form. This may be done by substituting

$$
\begin{aligned}
& \frac{A}{B}(\mathrm{I}+a) \text { for } \frac{A+x_{1}}{B+y_{1}}, \frac{A}{B}(\mathrm{I}+b) \text { for } \frac{\alpha+x_{2}}{\beta+y_{2}} \\
& \text { and } D \text { for } \frac{C}{Y} \frac{\beta+y_{2}}{\alpha+\beta+x_{2}+y_{2}} \text { which gives } \\
& \qquad X=Y \frac{A}{B}[\mathrm{I}+a+D(a-b)] .
\end{aligned}
$$

Equations (23) and (24) are the same except as to form and in what follows we shall refer to either indiscriminately as the fundamental equation of the Thomson bridge. It will be seen that here $a$ is the difference, in proportional parts, between the main ratio, including the connecting resistances to the branch points of the conductors, and the ratio $A / B$; that $a-b$ is the difference, in proportional parts, between the main and auxiliary ratios; and $D$ is a quantity usually small in comparison with unity.

\section{THE CORRECTION TERMS}

In the article referred to above it is pointed out that if the main and auxiliary ratios $\left(A+x_{1}\right) /\left(B+y_{1}\right)$ and $\left(\alpha+x_{2}\right) /\left(\beta+y_{2}\right)$ are made nearly equal and if the resistance $C$ is small in comparison with the resistances $X$ and $Y$, the sum of the second and third terms, or $D(a-b)$, is small and in many cases entirely negligible. 
Reeves ${ }^{14}$ has shown how the bridge may be balanced and at the same time the main and auxiliary ratios be adjusted to equality. His method consists in (a) adjusting the main ratio to give a zero current in the galvanometer; (b). removing the connector $Z$ thus making a simple bridge and adjusting the ratio

$$
\left(X+x_{2}+\alpha\right) /\left(Y+y_{2}+\beta\right) \text { equal to }\left(A+x_{1}\right) /\left(B+y_{1}\right) ;
$$

(c) restoring the connector $Z$ and again establishing a balance by adjusting the main ratio. While both the second and third adjustments disturb those made before, yet the result is that the ratios are more nearly equal than before the adjustments were made and consequently the correction term is smaller. Repeating the adjustments in the same order makes the correction term still smaller. Thus, by successive approximations the three ratios

$$
X / Y,\left(A+x_{1}\right) /\left(B+y_{1}\right) \text {, and }\left(\alpha+x_{2}\right) /\left(\beta+y_{2}\right)
$$

are made more and more nearly equal until there is no change in the balance on removing the connector, or the correction term $D$ $(a-b)$ is so small that no error is introduced on considering it zero.

This method was used by Dr. F. A. Wolff in the office of weights and measures in the Coast and Geodetic Survey prior to I90 I and has since been in use in the Bureau of Standards ${ }^{15}$ and in the national laboratories of Germany ${ }^{16}$ and England. ${ }^{17}$ Unless the resistance $C$ is small in comparison with $X+Y$ it is necessary to repeat the adjustment several times before a sufficiently close approximation is reached to neglect the correction term. In the case of very low resistances the number of successive approximations required is large and the process tedious. The time required for making the adjustments is materially reduced if the dials and switches for adjusting the main and auxiliary ratios are mechanically connected so that they can be easily changed by the same amount. With this arrangement the value of the correction term

\footnotetext{
${ }_{14}$ Proc. Phys. Soc. London, 14, p. I66; 1896. See also Fleming, Hd. Book. 1, p. 276; rgor; and N. F. Smith, Phys. Rev., 28, p. II3; Ig09.

15 Lloyd: Proc. Eng. Soc. W. Pa., 19, p. 403; I903.

${ }^{16}$ Jaeger, Lindeck, and Diesselhorst: $Z$ s. Instk., 23, p. $Z_{5} ; 1903$.

${ }_{17}$ Smith: Electrician, 57, p. Iorr; I906.
} 
is independent of the setting of the dials. With additional variable resistances in the auxiliary ratio the connector $Z$ may be removed and the two ratios set to an approximate equality, making the correction term negligible. Then with the connector replaced the balance may be made keeping the two ratios equal. Another alternative is to make the final adjustment by a variation of the resistance $X$ or $Y$. In some cases small changes in $X$ or $Y$ may be made by shunting them with a comparatively high resistance, the value of which need not be known to a high accuracy. In other cases the adjustment is made by sliding one of the potential connectors along the conductor. These different ways of making the necessary adjustments of the resistance are in more or less common use. In general, however, the use of variable ratios, the dial switches of which are mechanically connected, is found to be more convenient and reliable.

The resistances $x_{1}$ and $y_{1}$ while small in comparison with $A$ and $B$ are often large enough to introduce appreciable errors unless the proper corrections are made. It is necessary, therefore, to make some auxiliary measurements for determining the term $a$ which is the difference in proportional parts between $\left(A+x_{1} / B+y_{1}\right)$ and $A / B$. Where the main ratio can be varied in known small steps without changing the auxiliary ratio it is sufficient, with the connector $Z$ removed, to observe the change in the main ratio necessary to reestablish the balance on shifting ${ }^{18}$ the battery connections from $m$ and $m^{\prime}$ to $n$ and $n^{\prime}$. If this change in proportional parts be denoted by $w$, it is readily shown that to the required accuracy,

$$
a=\frac{\alpha}{\alpha+A} w \text {. }
$$

Where the dials of the two ratio sets are mechanically connected, this difference may be determined from the galvanometer deflections. This requires a determination of the sensitivity in addition to the change in deflection on shifting the connections from $m$ and $m^{\prime}$ to $n$ and $n^{\prime}$. From the sensitivity and the deflection the magnitude of $a$ is readily calculated. However, $a$ may be either positive or negative and it often requires more time to

${ }^{18}$ Lloyd: Proc. Eng. Soc. W. Pa., 19, p. 403; I903. Smith: Electrician, 57, p. roo9; I906. 
determine the sign than the magnitude. All things considered, the time and attention necessary for determining this correction $a$ is often as much as or more than that required for determining the correction term $D(a-b)$ or making the adjustments which make it negligibly small.

There is, therefore, in many cases as much as or more reason for carrying out a simple adjustment which makes the correction term, $a$, negligibly small as for carrying out an adjustment which makes the correction term, $D(a-b)$; negligibly small. What is really wanted is an adjustment which not only makes $b$ very nearly equal to $a$ but makes both very nearly equal to zero.

Jaeger and Diesselhorst ${ }^{19}$ recognized that it would be well to make such an adjustment and devised a means for carrying it out. The special feature of their method is the transfer of the connector $Z$ (see Fig. 7) from the terminals $k$ and $k^{\prime}$ to $n$ and $n^{\prime}$ and then balancing the bridge by an adjustment of $x_{1}$ or $y_{1}$. This gives

$$
x_{1} / y_{1}=X / Y \text { approximately, }
$$

and this makes $a$ very small if $x_{1}$ and $y_{1}$ are small in comparison with $A$ and $B$, since in the final adjustment $\left(A+x_{1}\right) / B+y_{1}$ is made equal to $X / Y$. While the method is good, the apparatus which they used was not well adapted to the purpose, so that in making the measurements it was necessary to make a number of successive approximations.

\section{ADJUSTMENTS MAKING CORRECTION TERMS SMALL}

When the main balance is established by an adjustment of the ratios, there is a decided advantage in having the dial switches of the main and auxiliary ratios mechanically connected. If, then, both $a$ and $b$ are to be made negligibly small, some means must be provided for an independent adjustment of the ratios $x_{1} / y_{1}$ and $x_{2} / y_{2}$. This can be accomplished by the use of variable low resistances forming a part of the resistances $x_{1}$ or $y_{1}$ and $x_{2}$ or $y_{2}$.

A variable low ${ }^{20}$ resistance developed jointly by J. H. Dellinger and the author is shown in Fig. 8 in section. Here the letters have

19 Tiss. Abh. d. P. T. R., 4, p. II9; I904.

${ }^{20}$ Phy. Rev., 32, p. 614; I9II. 33, p. 215; I9II. 
the following significance: $a$, a hard rubber tube; $b$, mercury; $c$ and $c_{1}$, copper terminals amalgamated where they come in contact with the mercury; $d$, an amalgamated copper rod; $e$, a spring clamp for holding the copper rod in position. Electrical connection is made through the terminal blocks $c$ and $c_{1}$, and the resistance is varied by changing the position of the copper rod. If the tube is made I $2 \mathrm{~cm}$ long and has a bore of $3-\mathrm{mm}$ diameter, the range is about o.or ohm, and the adjustment can easily be made to $0.00005 \mathrm{ohm}$. If a larger bore is used, closer adjustment may be obtained, though, of course, the range is reduced. Where a larger range is desired, it can be obtained by reducing the bore. Experience has shown, however, that it is not desirable to reduce the bore to less than I $\mathrm{mm}$ diameter. This gives a range, for a $\mathrm{I} 2-\mathrm{cm}$ tube, of about o.I ohm. In a number of measurements, especially with alternating currents, it is not necessary to know the resistances accurately, yet it is necessary to establish accurately some particular relation between them. In most of such cases these variable resistances make the adjustment a very simple matter. With one of these variable resistances on one side of each ratio and a small resistance on the other side, $a$ and $b$ may be made as small as we please. Obviously, if we desire to make such adjustments, we must begin by making a small rather than making $b$ equal to $a$ as has generally been done in measurements of high precision. If we begin by making $b$ equal to $a$, then any adjustment of $a$ is impracticable, since it disturbs the equality of $a$ and $b$. It is in consequence of this that it has been customary

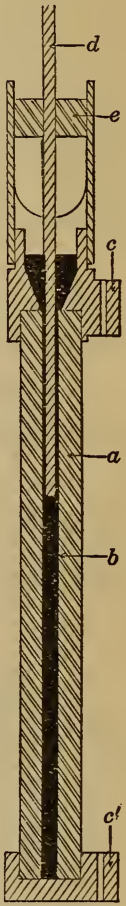

Fig. 8 to make some auxiliary measurement in order to determine the eorrection $a$.

The procedure which is now being followed in most of the precision resistance measurements at the Bureau of Standards, and which results in making both $a$ and $D(a-b)$ negligibly small, is as follows: Starting with the ratio $A / B, \alpha / \beta$ and $X / Y$ approximately equal

(I) With $n$ and $n^{\prime}$ as points of connection, the bridge is balanced by an adjustment of $x_{1}$ or $y_{1}$. $56 \mathrm{rog}^{\circ}-\mathrm{r} 2-\mathrm{ro}$ 
(2) With $m$ and $m^{\prime}$. as points of connection and the connector $Z$ between $X$ and $Y$ removed, the bridge is balanced by an adjustment of $x_{2}$ or $y_{2}$.

(3) With $m$ and $m^{\prime}$ as points of connection and the connection $Z$ between $X$ and $Y$ restored, the bridge is balanced by a proportional adjustment of both the main and auxiliary ratios, or in some cases by an adjustment of $Y$.

Where the connecting resistances are small in comparison with the ratio resistances and starting with the three ratios $X / Y$, $A / B$, and $\alpha / \beta$ approximately equal, the first operation makes $a$ negligibly small, the second makes $b$ equal to $a$, and the third fulfills the conditions of the fundamental equation. The three adjustments then give

$$
X=Y A / B
$$

which is the equation for the corresponding simple bridge.

Where the connecting resistances are large, the three adjustments are not entirely independent, since any change in making the second or third disturbs the first. It may, therefore, be necessary in some cases to repeat the adjustments in the same order, using those already made as first approximations.

\section{AN ILLUSTRATIVE PROBLEM}

To' illustrate the way in which the adjustments work out we shall consider the particular case of the comparison of $0.00 \mathrm{I}-\mathrm{ohm}$ standards, using the bridge regularly used in the comparison of standards designed to carry large currents.

In comparison of standards of the same denomination the ratio sets used with this bridge each have a total resistance of $200 \mathrm{ohms}$ and a ratio of 0.998 approximately. The higher side of each ratio is provided with a shunt whose resistance can be varied by means of four dials from a small value up to I oo ooo ohms. By changing the settings of the dials the ratios may be varied from 0.999 up to I.Oor, or higher if necessary. The dials on the two shunts operate together, so that the two ratios always have the same setting. As used, one end of each ratio set is connected to the potential terminals of one of the low resistances through a wire having a resistance of about $0.005 \mathrm{ohm}$, while the other end is connected 
through a variable mercury resistance to the potential terminals of the other low resistance. The wires form the major part of the resistances $y_{1}$ and $y_{2}$ and the variable mercury resistances the major part of the resistances $x_{1}$ and $x_{2}$, shown in Fig. 7 .

In the comparison of $0.00 \mathrm{I}-\mathrm{ohm}$ standards the following values chosen as an illustration are not only possible but are as probable as any others we might choose:

$$
\begin{aligned}
Y & =0.001 \mathrm{ohm} \\
X & =0.001001 \mathrm{ohm} \\
y_{1} & =0.005 \mathrm{ohm} \\
y_{2} & =0.005 \mathrm{ohm} \\
A & =100 \mathrm{ohms} \\
\alpha & =100 \mathrm{ohms} \\
C & =0.001 \mathrm{ohm}
\end{aligned}
$$

It follows, then, that for the three ratios $X / Y, A+x_{1} / B+y_{1}$, and $\alpha+x_{2} / \beta+y_{2}$ to be equal, or $a=b=0$ we must have

$$
\begin{aligned}
B & =99.9 \\
\beta & =99.9 \\
x_{2} & =0.005005 \\
x_{1} & =0.005005
\end{aligned}
$$

But on beginning the adjustments we do not know the exact ratio of $X$ to $Y$ nor of $x_{2}$ to $y_{2}$, nor what should be the ratio of $A$ to $B$. As the resistances to be compared are generally adjusted to within o.I per cent of their nominal value, $A$ and $B$ have values to begin with which may differ by not more than 0.2 per cent from the values they should have and will have after the final adjustment. Also, $x_{2}$ has a value which may differ by $0.005 \mathrm{ohm}$ from the value it will have after adjustment.

Let us assume, then, that

$$
\begin{aligned}
B & =\text { IOO.I } \\
\alpha & =\text { IOO.I } \\
\text { and } x_{2} & =0.00 \mathrm{I}
\end{aligned}
$$

It should be observed that the values assumed are such that the lack of adjustment of the ratios and $x_{2}$ cause errors in the same direction in the adjustment of $x_{1}$.

I. With these values for $B, \beta$ and $x_{2}$ and the values given above for the other resistances, with the connector $Z$ in place and $n$ and $n^{\prime}$ as 
branch points adjusting $x_{1}$ so as to balance the bridge necessitates making $x_{1}=0.004950 \mathrm{ohm}$. This differs from the value which it should have as given above by $0.00005 \mathrm{ohm}$, which in comparison with 100 ohms is 5 parts in 10000000 . In this case, then, the first adjustment leaves $a=-0.0000005$, which is negligible in most if not quite all resistance measurements.

2. With the connector $Z$ removed and using $m$ and $m^{\prime}$ as branch points, adjusting $x_{2}$ so as to balance the bridge makes the ratios $\left(A+x_{1}\right) /\left(B+y_{1}\right)$ and $\left(\alpha+x_{2}\right) /\left(\beta+y_{2}\right)$ equal, to the precision with which the balance is established. In this adjustment $b$ is easily made equal to $a$ to well within the limits required for the most precise measurements. However, there is a possibilty that this equality may be disturbed in adjusting the main and auxiliary ratios.

3. With the connector $Z$ in place and using $\dot{m}$ and $m^{\prime}$ as branch points an equal adjustment of $A$ and $\alpha$ so as to balance the bridge still leaves $\left(A+x_{1}\right) /\left(B+y_{1}\right)$ and $\left(\alpha+x_{2}\right) /\left(\beta+y_{2}\right)$ at least very nearly equal since $x_{1}$ and $y_{1}$, and $x_{2}$ and $y_{2}$ are very nearly equal and small in comparison with $A, \alpha, B$, and $\beta$. As this last adjustment can not ordinarily be made to a precision better than I part in I ooo ooo the three adjustments give

$$
X=Y A / B
$$

to as high a precision as can be obtained in the final balance of the bridge.

For convenience in carrying out the adjustments it is sometimes desirable to have two keys and two rheostats in the battery circuit. Key No. I closes the circuit through the bridge and the rheostat having the higher resistance, while key No. 2 closes the circuit through the bridge and the rheostat having the lower resistance. Key No. 2 is used in making the adjustment (2) while key No. I is used in making the adjustment (3). For making the adjustment (I) key No. I is generally used, though in most cases No. 2 could be used without a change in the setting of the rheostat. As some of the measurements are actually carried out a single key is used for making the three adjustments and no change is made in the setting of the rheostat. 
Had the ratio coils been Io instead of 100 ohms, the first adjustment would have left $a$ about ten times as large and for the most precise measurements would have necessitated repeating the adjustments in the same order. In conductivity measurements of samples in the form of rods or wires the ratio of the resistances of the conductors may not be known even approximately. In such cases it is better to make an approximate adjustment using $m$ and $m^{\prime}$ as branch points before taking up the adjustments in order. Even then if the adjustment 3 requires much of a change in the ratio $A / B$ the adjustments should be repeated. If, on the other hand, the change necessary in this ratio is small, we know that we need make no further adjustments.

Where the adjustments are made in this way the accuracy is as high or higher than where any of the eonnecting resistances are determined and the corrections are applied, while the time and attention necessary for making the adjustments are generally considerably less.

\section{SENSITIVITY WITH D'ARSONVAL GALVANOMETER}

In most of the work at the Bureau of Standards with the Thomson and Wheatstone bridges, D'Arsonval galvanometers of high sensitivity are used. These galvanometers are so designed and constructed that the motion of the moving system is critically damped with a comparatively low resistance in the external circuit, in some cases as low as 35 ohms. Their operation is so much more satisfactory when critically damped that adjustment to this condition is regularly made.

It is easily seen that if the bridge is out of balance by a small amount $d$, and the galvanometer is not connected, the difference in potential between the points to which it is to be connected is

$$
d \sqrt{P X} Y /(X+Y) \text {. }
$$

Here $P$ is the power dissipated in the conductor $X$, and $d$ is the equivalent of $\Delta X / X$, where $\Delta X$ is the amount by which if $X$ were changed the bridge would be balanced. If in adjusting to a balance proportional changes are made in $A$ and $\alpha$ or in $B$ and $\beta$, then $d=-\Delta A / A$ or $\Delta B / B$. 
(a) If the resistance of the bridge between the terminals to which the galvanometer is to be connected is less than the external critical resistance of the galvanometer, then some additional resistance must be connected in series to make up the deficit. Under these conditions the deflection of the galvanometer is

$$
S_{e} d \sqrt{P X} Y /(X+Y)
$$

or the sensitivity $S$, the ratio of the deflection to the lack of balance of the bridge, is given by the equation

$$
S=S_{e} \sqrt{P X} Y /(X+Y)
$$

when $S_{e}$ is the voltage sensitivity of the galvanometer under the critically damped condition. This equation and the others which will be given were derived from a consideration of the relations which exist in the Thomson bridge. However, they give the sensitivity not only of the double bridge but also of the simple bridge and the multiple bridge.

(b) If the resistance of the bridge is larger than the external critical resistance of the galvanometer, then some resistance must be placed in parallel with the galvanometer, otherwise the motion of the moving system is underdamped. As the resistance is larger than in the previous case, the current for a given emf is less. Also a part of the current passes through the shunt instead of through the galvanometer. As a result the deflection is less than in the former case by the factor $r_{c} / r_{b}$, where $r_{c}$ is the external critical resistance of the galvanometer and $r_{b}$ is the resistance of the bridge. Therefore

$$
S^{\prime}=S_{e} \sqrt{P X} Y /(X+Y) r_{c} / r_{b}
$$

where $S^{\prime}$ is the sensitivity in case it is necessary to shunt the galvanometer.

(c) If changes in temperature are to be measured by changes in the resistance, $X$ and if $\alpha$ is the proportional change in $X$ per degree $_{c}$, the sensitivity $S_{t}$ of the combination of resistance thermometer, bridge, and galvanometer is given by the equation

or

$$
\begin{aligned}
& S_{t}=\alpha S_{e} \sqrt{P X} Y /(X+Y) \\
& S_{t}=\alpha S_{e} \sqrt{P X} Y /(X+Y) r_{c} / r_{b}
\end{aligned}
$$


depending on whether $r_{b}$ is less or more than $r_{c}$. These equations $(28,29$, and 30$)$ show that if the resistance of the bridge is less than the external critical resistance of the galvanometer the sensitivity is directly proportional to the voltage sensitivity of the galvanometer, the square root of the power dissipated in the conductor times the square root of its resistance, and the ratio $Y /(X+Y)$. If the resistance of the bridge is more than the external critical resistance of the galvanometer, the sensitivity is reduced by the factor $r_{c} / r_{b}$.

It will thus be seen that the voltage sensitivity and the external critical resistance of the galvanometer are matters of first importance in fixing the sensitivity of the system. There are also other constants of the galvanometer which have some effect upon the precision which may be obtained. The more important of these are the period of the moving system and the resolving power of the optical system. We know, for example, that under ordinary conditions if the galvanometer has a period of 20 seconds we are not able to obtain as high a precision as with a galvanometer having the same sensitivity but a period of only 3 seconds. On the other hand, we can not state that the precision is inversely proportional to the period or depends in any definite way upon the period. Likewise, we can not state definitely the way in which other constants affect the precision. However, the voltage sensitivity and the external critical resistances are by far the more important constants, and their effect upon both the sensitivity of the system and the precision which may be obtained is definite and known. In galvanometers having a fairly large restoring moment or "stiff" suspensions, the effect of particular changes in the design such as increase in the diameter of the wire used in winding the coil, an increase in the field strength, etc., can be calculated from the theory. On the other hand, in D'Arsonval galvanometers of the highest sensitivity the moment of restoration is so small and the intensity of the field is so large that the magnetic impurities in the coil, even where every precaution has been taken to exclude them, have a marked influence on the behavior of the instrument. On account of these impurities one of two instruments, constructed as near alike as possible, may easily have two or three times the sen- 
sibility of the other. Consequently an instrument maker in carrying out a particular design can not produce a galvanometer having just the constants which, according to the theory, we should have expected. Instead of trying to get a galvanometer having particular constants we should select the best galvanometer available for the work and then design the rest of the apparatus so the system will have its maximum sensitivity for the particular galvanometer. If, on the other hand, the bridge is designed first, generally no galvanometer can be obtained which will work to its best advantage with the particular bridge.

To obtain the highest sensitivity the power dissipated in $X$ should be as large as possible, without the uncertainties in its resistance, on account of the heating by the test current, becoming equal to the precision sought. That the test current may not be limited by the heating in some other part of the bridge the various resistances should be so designed that their proportional changes, on account of heating by the part of the current which they carry, is less than the proportional change in $X$. Further, the resistance of the ratio coils should be large, so that the effects of the connecting resistances will be small and the various adjustments easily made. On the other hand, the resistance between the branch points to which the galvanometer is connected should preferably be less than the external critical resistance of the galvanometer, so it need not be shunted, thus reducing the sensitivity of the system. Ordinarily, the resistances of the ratio coils should be so chosen that the galvanometer may. be critically damped by putting only a few ohms in series.

The conductor $X$ should be so designed that the proportional change in resistance will be small for a given current. Also the resistance $X$ should be comparatively large. Generally, however, the conductor is already constructed so we can neither change the load which it will carry nor the value of its resistance. In most cases, too, either the ratio $X / Y$ is fixed by other considerations or the test current which may be used is limited on account of the heating which it may produce in $Y$. It will thus be seen that of the various factors which affect the sensitivity we are generally at liberty to change only a few. In comparing resistance standards of low value there is seldom any difficulty about the heating 
of the ratio coils by the part of the test current which they carry. Therefore nearly everything which may be done toward getting a high sensitivity is done when the best galvanometer available, considering mainly its voltage sensitivity and external critical resistance, is selected and the resistance of the bridge between the galvanometer connectors is made only slightly less than the external critical resistance of the galvanometer.

However, the sensitivity of the system is not the only factor which has an effect upon the precision which may be obtained. Reference has already been made to the period of the galvanometer and the resolving power of its optical system. Of the other things which should be mentioned the thermoelectric effects are probably of the most importance. Under ordinary conditions the galvanometer circuit may be expected to have a thermoelectromotive force which is almost always changing and may easily amount to a few microvolts. Since to get the precision desired often requires the detection of a hundredth of a microvolt, it is necessary to work with a "false zero;" that is, the bridge is considered balanced when with the galvanometer circuit closed there is no change in the deflection following the closing or opening of the battery circuit. What is better than this is the reversal of the test current, since for the same lack of balance the change in deflection is twice as large. If the thermoelectromotive forces are changing rapidly, they limit the precision of the balance even when made in this way. Trouble on this account can be considerably reduced by keeping as much of the galvanometer circuit as possible in a well stirred oil bath. The rest of the circuit should be made up as far as possible from a homogeneous conductor. All loose connections should be avoided, since the surfaces in contact are usually oxidized and so have a high thermal resistance. If, then, there is a flow of heat across the connection, there is a high temperature gradient in the oxide and so the two junctions between the oxide and metal may be at different temperatures, in which case there is in general a thermoelectromotive force.

Where the balance is made using a false zero or a closed galvanometer circuit the unbalanced inductances in the bridge are often troublesome and may limit the precision of the balance. 
It will thus be seen that the expressions derived for the sensitivity do not contain all the factors having an effect on the precision which may be obtained. It is believed, however, that with the apparatus used and with the conditions under which the measurements are carried out these expressions contain the more important factors and all the factors whose effects can be definitely stated. Investigators working with different apparatus and under different conditions have found the precision attainable limited in different ways. In deriving expressions for the sensitivity ${ }^{21}$ they have therefore included different factors. For example, at one time Daniell cells were used as the source of the test current and as their resistance limited the value of the current it is not uncommon to find expressions for the sensitivity which contain a term representing the resistance of the battery. Thomson, ${ }^{22}$ in 1862 , called attention to the fact that the heating of the conductor by the test current is one of the important factors in limiting the precision which may be attained in the measurement. However, a number of papers appeared later in which there is no reference to the heating and it was not until I 895 that Schuster ${ }^{23}$ again called attention to it and derived expressions for the sensitivity based on the current which the conductor will carry with the permissible rise in temperature.

In work with resistance thermometers the compensation for the changes in the resistance of the leads is not always easily accomplished to the precision desired. For this reason some resistance thermometers are being made with current and potential terminals and have the branch points so located that under the conditions of use all parts of the conductors between them are to be at the same temperature. The resistance between the branch points is generally several ohms and the resistance of the connectors from a few hundredths to a tenth ohm. On account of the comparatively high resistance, the high temperature

21 The following is a partial list of the papers in which the sensitivity is discussed:

Schwendler: Phil. Mag., 31, p. 364; r866. Heaviside: Phil. Mag., 45, p. I14; I873. T. Gray: Phil. Mag., 12, p. 283; I88r. Rayleigh: Proc. Roy. Soc., 49, p. 203; r89r. Schuster: Phil. Mrag., 39, p. I75; I895. Gray: Abs. Meas., 1, p. 331. Flemming: H. Book, 1, p. 233. Jaeger: Zs. f. Instk., 26, p. 69; I906. Calender: Proc. Phys. Soc., 22, p. 220; rgro.

22 Phil. Mag., 21, p. 149; r862.

${ }^{23}$ Phil. Mag., 39, p. I75; 1895. 
coefficient of the resistance material, and its small surface, the test current is limited to a very small value. Even rooo-ohm coils of the ordinary construction will carry more current without an appreciable change in resistance. Therefore, when such a conductor is connected into a Thomson bridge in the usual way, there is no reason why the battery and galvanometer connections may not be interchanged. With the usual connections the ratio coils make up a large part of the resistance between the galvanometer connectors. As a result the resistance of the ratio coils must be kept comparatively low or some sacrifice made in the sensitivity. On the other hand, with the connections reversed the resistance of the bridge depends almost entirely on the resistance $X+Y$. With this arrangement, therefore, the advantage of using ratio coils of high resistance may generally be obtained without a sacrifice in the sensitivity.

\section{THE MULTIPLE BRIDGE}

As the Thomson bridge is ordinarily used the galvanometer is connected to the points between the ratio coils, while in the case we have just been considering the battery was connected to these same points. Having used an auxiliary ratio set with good results first in the galvanometer circuit and then in the battery circuit, the next step naturally would be the use of auxiliary ratios in both the galvanometer and battery circuits. The author has mentioned this matter to different persons who have used the Thomson bridge in precision measurements and found that each one had considered it.

The connections for a bridge of this kind with three sets of auxiliary ratios are shown in Fig. 9. If adjustments are made so that there is no current through the galvanometer with the connectors $Z, Z_{1}$, and $Z_{2}$ in place and with them removed alternately it will easily be seen that

$$
X / Y=A / B
$$

Since in this case at least one set of auxiliary ratios must carry the test current, either the ratio set must be of low resistance or the test current must be small. The advantage of the use of an auxiliary ratio is largely lost unless its resistance is large in com- 
parison with the resistance of the conductor with which it may be considered in parallel (the resistance $Z$ referred to above, p. $58 \mathrm{r}$ ). The multiple bridge may therefore be considered as limited to

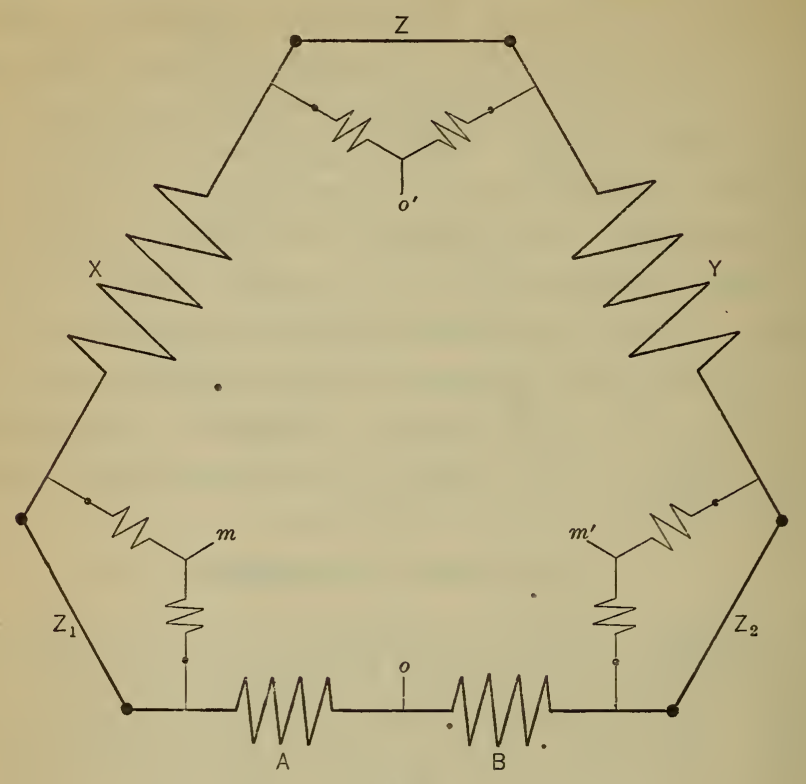

Fig. 9

that class of measurements in which a small test current is used, as in the case of resistance thermometers.

\section{THEORY WITH NONLINEAR CONDUCTORS}

Equation (23), on which the preceding discussion of the Thomson bridge is based, was derived for the case in which the fourterminal conductors are linear. ${ }^{24}$ We can not, therefore, assume that it is applicable in the more general case. In fact, if we will consider the simplest particular cases where the four-terminal conductors are not linear, we will see that this equation does not give the exact relations necessary for a zero current through the galvanometer. If, however, the ratio coils have a large resistance in comparison with the resistance of the four-terminal conductors, and if the cross resistances of the latter are small in comparison with their direct resistances, the errors introduced by using the

24 For explanation of the difference between linear and nonlinear conductors, see p. 57 x. 
equation will generally be so small that they need not be considered. In the limiting case where the ratio coils have a very high resistance, since they carry no appreciable part of the current, it is easily seen that the ordinary equation may be considered to give the exact relations between the different resistances as it does in the case of linear conductors. Searle ${ }^{25}$ has recently published equations giving the general relation between the resistances necessary for the zero current in the galvanometer. $\mathrm{He}$ also points out the fact that in the particular case in which the

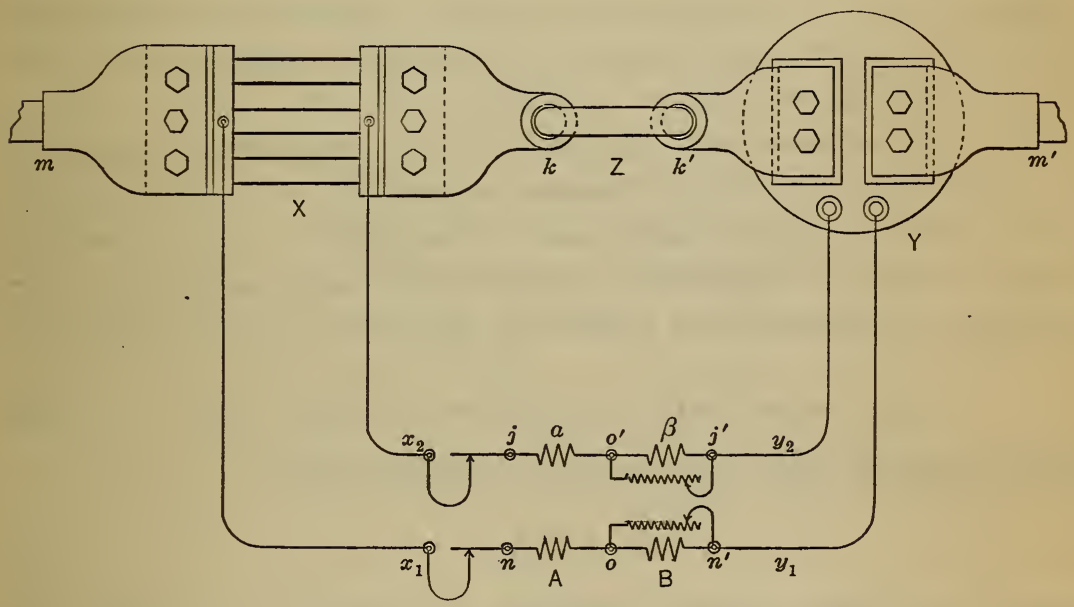

Fig. 10

bridge is balanced, both with the connector $Z$ in place and with it removed, the term or terms containing the cross resistance disappear.

Without going further into the theory we wish now to show that, whether the four-terminal conductors are linear or nonlinear, if the adjustments are carried out as outlined above (p. 585), the same simple relations exist between the resistances. To show this we shall consider the adjustments to have been carried to the point where with either $m$ and $m^{\prime}$ or $n$ and $n^{\prime}$ as battery connections (see Fig. Io) and with the connector $Z$ either in place or removed the current through the galvanometer, connected to $o$ and $o^{\prime}$, may be considered zero. It follows, then, from the recip- 
rocal theorem referred to above, that if $o$ and $o^{\prime}$ are used as battery connections $m$ and $m^{\prime}$ and $n$ and $n^{\prime}$ may be considered to be at the same potential, whether the connector $Z$ is in place or not. With the battery connected at $o$ and $o^{\prime}$ the conditions necessary for the terminals $m$ and $m^{\prime}$ and $n$ and $n^{\prime}$ to be at the some potential are that

$$
A / B=\left(x_{1}+X+x_{2}+\alpha\right) /\left(y_{1}+Y+y_{2}+\beta\right)
$$

and

$$
\left(A+x_{1}\right) /\left(B+y_{1}\right)=\left(X+x_{2}+\alpha\right) /\left(Y+y_{2}+\beta\right) .
$$

Here $X$ and $Y$ are the four-terminal resistances $k-m / q-n$ and $k^{\prime}-m^{\prime} / q^{\prime}-n^{\prime}$, while $x_{1}, x_{2}, y_{1}$, and $y_{2}$ are the three-terminal resistances $n-m / n-k, j-k / j-m, n^{\prime}-m^{\prime} / n^{\prime}-k^{\prime}$, and $j^{\prime}-k^{\prime} / j^{\prime}-m^{\prime}$.

The fact that removing the connector $Z$ produces no change in the difference in potential between $m$ and $m^{\prime}$ or $n$ and $n^{\prime}$ shows that it carries no appreciable part of the current, so the terminals $k$ and $k^{\prime}$ may be considered to be at the same potential. The condition necessary for $k$ and $k^{\prime}$ to be at the same potential is

$$
\left(A+x_{1}+X\right) /\left(B+y_{1}+Y\right)=\left(x_{2}+\alpha\right) /\left(y_{2}+\beta\right)
$$

From equations (39), (40), and (4I) it follows that

$$
X=Y A / B
$$

which is the same simple relation as was obtained when we were considering linear conductors. Where the four-terminal conductors are nonlinear the Thomson bridge constitutes a system which can hardly be said to be simple. It seems rather remarkable, therefore, that by a few simple adjustments we can obtain a relation between the resistances which is sufficiently accurate for use in the most precise measurements.

\section{THEORY USING ALTERNATING CURRENT}

The Thomson bridge has been used with alternating current by Sharp and Crawford ${ }^{26}$ in the comparison of inductances in heavy current resistances and by Barnett ${ }^{27}$ in the measurement of inductances and capacities. The equations used by these authors can, 
however, give accurate results only in special cases or in cases in which the Thomson bridge has little, if any, advantage over the ordinary bridge.

In discussing the comparison of the resistances of nonlinear conductors by means of the Thomson bridge, we found it desirable to consider only the case where certain auxiliary adjustments had been made. When we come to the consideration of the relations between the various resistances, inductances, and the frequency, when alternating current is used, auxiliary adjustments become of much more importance. A general relation between the various quantities affecting the balance of the bridge would necessarily be very complicated; for in addition to the two cross resistances (which are responsible for much of the complication when direct current is used), we should have to consider two cross inductances and various self and mutually induced electromotive forces caused by three components of the current, each of which differs in phase from the others. We shall, therefore, not consider the general problem but limit our discussion to the special case in which, in addition to the auxiliary resistance adjustments outlined above (p. 585) corresponding adjustments of the inductances are made and the parts of the bridge are so arranged that certain of the mutual inductances have a negligible effect upon the conditions of the balance.

Let us assume that adjustments have been carried out so that, with alternating current supplied either through the leads $m$ and $m^{\prime}$ (see Fig. II) or $n$ and $n^{\prime}$, and with the connector $Z$ either in place or removed, there is no current through a galvanometer connected to $o$ and $o^{\prime}$. We then have three independent balances of the bridge with alternating current which correspond to the three independent balances with direct current which we have considered above. Here the galvanometer leads must be brought out in such a way that the alternating test current can induce no emf in them.

With this adjustment, it follows from the reciprocal theorem (considered above, p. 563), that if the current supply is led in through the leads connected to $o$ and $o^{\prime}$, a galvanometer connected alternately to $m$ and $m^{\prime}$ and to $n$ and $n^{\prime}$ will indicate a 
zero current, if the galvanometer leads are located the same as the current leads were in making the adjustments. Now, as before, the connector $Z$ may be removed without disturbing the balance. This shows that with this connection it carries no appreciable part of the current.

If, then, a galvanometer were connected to the terminals $k$ and

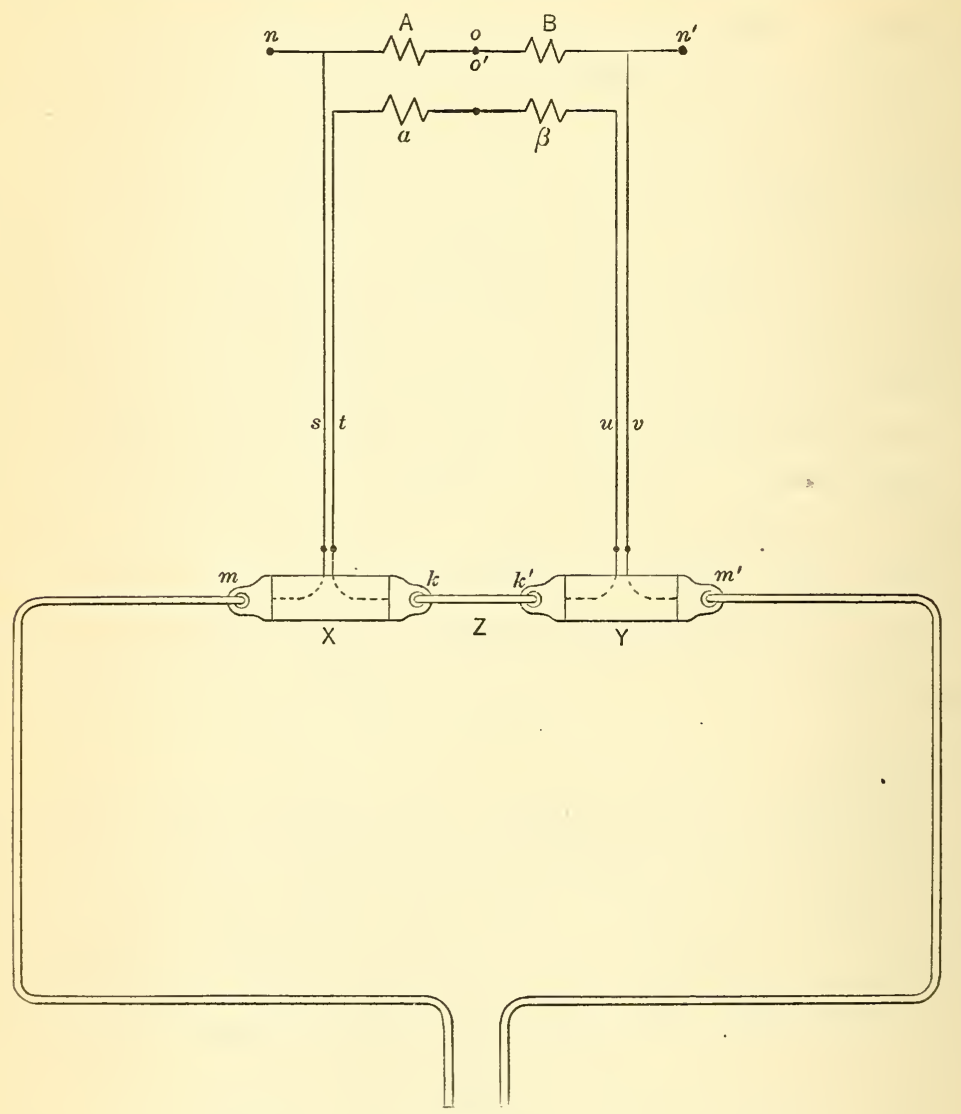

Fig. 11

$k^{\prime}$, it would indicate a zero current, providing the leads were brought out in such a way as to have the same mutual inductance with respect to the rest of the system as the connector $Z$, when in place.

The relations between the various quantities are therefore the same as in a simple bridge which is balanced when either of three 
pairs of potential connections are used. In this case we may consider the bridge as made up of two conductors in parallel, each of which has five terminals, $o, n, m, k$, and $o^{\prime}$, and $o, n^{\prime}, m^{\prime}$, $k^{\prime}$, and $o^{\prime}$. If no appreciable error is introduced on neglecting the mutual inductance between these two conductors-that is, between the two halves of the bridge-we have the following relations between the impedances:

$$
\begin{aligned}
& \frac{o-n / o-o^{\prime}+\iota p \overline{\left(o-n / o-o^{\prime}\right)}}{o-n^{\prime} / o-o^{\prime}+\iota p \overline{\left(o-n^{\prime} / o-o^{\prime}\right)}}=\frac{n-m / o-o^{\prime}+\iota p \overline{\left(n-m / o-o^{\prime}\right)}}{n^{\prime}-m^{\prime} / o-o^{\prime}+\iota p \overline{\left(n^{\prime}-m^{\prime} / o-o^{\prime}\right)}} \\
= & \frac{m-k / o-o^{\prime}+\iota p \overline{\left(m-k / o-o^{\prime}\right)}}{m^{\prime}-k^{\prime} / o-o^{\prime}+\iota p \overline{\left(m^{\prime}-k^{\prime} / o-o^{\prime}\right)}}=\frac{k-o^{\prime} / o-o^{\prime}+\iota p \overline{\left(k-o^{\prime} / o-o^{\prime}\right)}}{k^{\prime}-o^{\prime} / o-o^{\prime}+\iota p \overline{\left(k^{\prime}-o^{\prime} / o-o^{\prime}\right)}}
\end{aligned}
$$

Here $o-n / o-o^{\prime}, n-m / o-o^{\prime}, m-k / o-o^{\prime}$, and $k-o^{\prime} / o-o^{\prime}$ are the resistances and $\overline{o-n / o-o^{\prime}}, \overline{n-m / o-o}, \overline{m-k / o-o^{\prime}}$, and $\overline{k-o^{\prime} / o-o^{\prime}}$ are the inductances of the four-terminal conductors, $\iota$ is the square root of minus one and $p$ is $2 \pi$ times the frequency of the alternating current. The same system is used to designate the resistances and inductances of the right-hand side of the bridge. Since we are considering $o$ and $o^{\prime}$ as current terminals common to two conductors in parallel, the symbols do not have quite the same significance as that given above. (See p. 56r.)

If we may consider the mutual inductance between $A$ and the other parts of the system, including the lead $n$ as negligible, then $\overline{o-n / o-o^{\prime}}$ is the inductance $L_{A}$. Under the same conditions $\overline{o-n^{\prime} / o-o^{\prime}}$ is the inductance $L_{B}$. If we may consider the mutual inductance between $X$, with its leads $m$ and $Z$, and the rest of the system beyond the potential terminals as zero, then $\overline{m-k / o-o^{\prime}}$ is the inductance $L_{X}$, if the lead $m$ and the connector $Z$ are in the normal position of the current leads to the conductor $X$.

As both the resistance and inductance of $X$ will in most cases be very small, it is important that the mutual inductance between $X$, with its current leads and the rest of the system, should be very small, otherwise considering it zero may introduce an error. How. important it is that this mutual inductance be made very small will be understood when we consider that the resistance of the conductor $X$ may be $0.000 \mathrm{I}$ ohm or less and the current through $56109^{\circ}-\mathrm{I} 2--\mathrm{II}$ 
it may be 1000 amperes or more. With an alternating current of this magnitude the mutual inductance does not need to be large for the induced emf to be appreciable in comparison with o.I volt, the difference in potential between the potential terminals. With the arrangement shown in Fig. I , even when the ratio sets are at a considerable distance from the four terminal conductors, the emf induced in the ratio coil $A$ is very appreciable in comparison with the drop in potential in the low resistances. Unless this emf is balanced by an equal emf, induced either in the auxiliary ratio arm $\alpha$ or in the other main ratio arm, an error will be introduced. The magnitude of this error may be of the order of the difference of the induced emf in $A$ and $\alpha$ divided by the voltage across the potential terminals of $X$, though generally it will be considerably less.

If care is taken to place $A$ and $\alpha$ at a considerable distance from the conductors which carry the large current and in such positions that they have very nearly the same mutual inductance with respect to that part of the system which carries the large current, and if the potential leads are placed near each other or are twisted together, the mutual inductance between $X$, with its current leads and that part of the left side of the bridge beyond the potential terminals, may be considered zero.

Under the conditions similar to those just considered $\overline{m^{\prime}-k^{\prime} / o-o^{\prime}}$ is the inductance $L_{Y}$. As a matter of convenience we shall call $\overline{n-m / o-o^{\prime}}, \overline{k-o^{\prime} / o-o^{\prime}}, \overline{n^{\prime}-m^{\prime} / o-o^{\prime}}$, and $\overline{k^{\prime}-o^{\prime} / o-o^{\prime}}$ the inductances $l_{s}$, $l_{t}, l_{u}$, and $l_{v}$. Making these substitutions in equation (36) we have

$$
\begin{gathered}
\frac{A+\iota p L_{A}}{B+\iota p L_{B}}=\frac{s+\iota p l_{s}}{v+\iota p l_{v}} \\
\frac{A+\iota p L_{A}}{B+\iota p L_{B}}=\frac{X+\iota p L_{X}}{Y+\iota p L_{Y}} \\
\frac{A+\iota p L_{A}}{B+\iota p L_{B}}=\frac{t+\alpha+\iota p\left(l_{t}+l_{a}\right)}{u+\beta+\iota p\left(l_{u}+l_{\beta}\right)}
\end{gathered}
$$

If the bridge is balanced under the conditions given above, all of these equations are satisfied. It is, however, the second which gives the relations between the quantities which we wish to com- 
pare and with which we are most concerned. This equation may be put in the following form

$$
\begin{aligned}
& A Y-p^{2} L_{A} L_{B}+\iota p\left(A L_{Y}+Y L_{A}\right)= \\
& B X-p^{2} L_{B} L_{X}+\iota p\left(B L_{X}+X L_{B}\right)
\end{aligned}
$$

or separating the real and imaginary parts we have

$$
A Y-B X=p^{2}\left(L_{A} L_{Y}-L_{B} L_{X}\right)
$$

and

$$
A L_{Y}+Y L_{A}=B L_{X}+X L_{B}
$$

These equations show that where the time constants are small the balance of the bridge is practically, independent of the frequency. Inspection will show that if $A$ and $B$ or $A$ and $X$ or $B$ and $Y$ can be considered to have equal time constants, the balance of the bridge is independent of the frequency except in so far as the resistances and inductances may themselves be functions of the frequency. Low-resistance standards suitable for use in alternating current measurements, such as we are concerned with here, necessarily have small time constants. If, then, the ratio coils $A$ and $B$ have small time constants and the frequency is not high, it will be convenient to write equation (4I) in the following slightly different form

$$
X=\frac{A}{B} Y\left[\mathrm{I}+\frac{p^{2} B}{A Y}\left(L_{B} L_{X}-L_{A} L_{Y}\right)\right]
$$

Where $\frac{p^{2} B}{A Y}\left(L_{B} L_{X}-L_{A} L_{Y}\right)$ is small and may be looked upon as a correction term. We, therefore, have approximately

$$
X=\frac{A}{B} Y
$$

and

$$
\frac{L_{X}}{X}=\frac{L_{Y}}{Y}+\frac{L_{A}}{A}-\frac{L_{B}}{B}
$$

To show that no errors need be introduced by the use of these approximate equations, we may consider the following example 


$$
\begin{array}{ll} 
& p=2 \pi \times 60 \\
A=\text { Io ohms } & L_{A}=4 \text { microhenrys } \\
B=\text { Io ohms } & L_{B}=-3 \text { microhenrys } \\
Y=\text { o.00I ohm } & L_{Y}=0.005 \text { microhenry } \\
X=\text { ? ohm } & L_{X}=\text { ? microhenry }
\end{array}
$$

From equation (45) we have

$$
\frac{L_{X}}{X}=(5+0.04+0.03) \times 10^{-6} \text { seconds }
$$

and since we know that $X$ must be very nearly $0.00 \mathrm{I}$ ohm $L_{x}=$ 0.00507 microhenry.

This value substituted in (43) shows that the correction term is less than $\mathrm{IO}^{-6}$, a quantity which is negligible even in very precise resistance measurements. We may, therefore, consider $X$ exactly $0.001 \mathrm{ohm}$, in which case $L_{x}$ may be considered exactly 0.00507 microhenries. Since ratio coils can be obtained having time constants of only one-tenth the values used in the example and since we shall seldom be concerned with low resistance standards having so large a time constant the approximate equations (44) and (45) can in most cases be used, instead of the exact equations (4I) and (42).

We have seen that when the bridge is balanced under each of the three conditions given above and when we can neglect the effect of certain of the mutual inductances, we have fairly simple relations between the resistances, inductances, and frequency, and if the time constants of all the conductors are small, the relations are very simple.

To bring about these relations it is necessary that at least three of the eight resistances and three of the eight inductances be variable. That is, to satisfy equations (37), (38), and (39) at least one resistance and one inductance in each must be variable either continuously or in small steps, over a range corresponding to the range in values of the resistance and inductance of the standards to be compared while using a particular auxiliary standard $Y$ and particular ratio coils $A$ and $B$. If the time con- 
stants of the main ratio coils are small and nearly equal, we may consider equation (37) and (39) satisfied when $\frac{A+s}{B+v}$ and $\frac{\alpha+t}{\beta+u}$ can be considered as equal to $A / B, \frac{p l_{s}}{A+s}$ can be considered equal to $\frac{p l_{v}}{B+v}$, and $\frac{p\left(l_{t}+l_{\alpha}\right)}{t+\alpha}$ can be considered equal to $\frac{\left(p l_{\beta}+l_{u}\right)}{\beta+u}$. These relations between the resistances being the same as those which we have considered above when using direct current, similar apparatus may be employed in making the adjustments.

As we have assumed the time constants of the ratio coils to be small and nearly equal, we can not change either as a part of the adjustments necessary in establishing the triple balance of the bridge. This makes it necessary to provide some means for adjusting the time constant of conductor $X$ or $Y$ as well as of $s$ or $v$, and $t$ (or $\alpha$ ) or $u$ (or $\beta$ ). A convenient way of providing for an adjustment equivalent to an adjustment of the time constant of the conductor $Y$ is to place a movable coil in one of the potential leads, as shown in Fig. I 2 , in such a way as to introduce a variable mutual inductance $M$ between the potential lead and one of the current leads. When this mutual inductance is taken into consideration we have $L_{Y}+M$ in place of $L_{Y}$ in all the above equations. The relation between the time constants of the four terminal conductors and the main ratio coils is then

$$
\frac{L_{X}}{X}=\frac{L_{Y}+M}{Y}+\frac{L_{A}}{A}-\frac{L_{B}}{B} \text {. }
$$

A variable mutual inductance suitable for use in the comparison of low resistance standards may consist of a coil of 8 to ro turns, 8 to ro centimeters in diameter, and one of the current leads with a suitable support for holding them in the desired relative positions. The mutual inductance may be varied either by rotating the coil or by changing its distance from the current lead. With the former the inductance may be varied continuously from the maximum positive value to a corresponding negative value. It is also the more convenient. In some cases the inductance may be determined by calculation from the dimensions or in general by comparison with other inductances, either self or mutual. The 
calibration need not be made to a high accuracy since the time constants to be compared are so small that we shall seldom be concerned with more than two significant figures.

The coil for the mutual inductance $M$ necessarily changes the inductance $l_{v}$. Therefore, to make $\frac{l_{s}}{s}=\frac{l_{v}}{v}$ it is necessary to put a coil of corresponding self inductance in the conductor $s$. This coil must be so placed that the mutual inductance between it and the conductor which carries the main part of the test current is

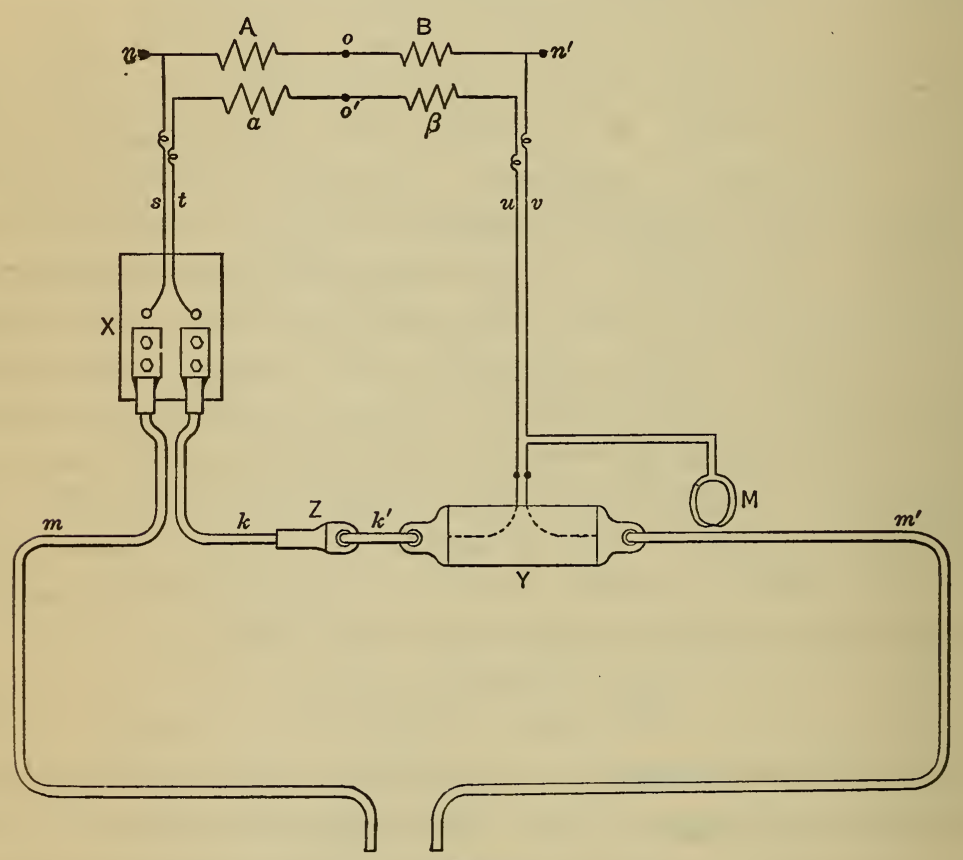

Fig. 12

negligibly small. If this coil is made in two sections, the changes in its inductance necessary for balancing the bridge may be made by changing their relative position. If the auxiliary ratio coils have very nearly equal time constants, the little adjustment necessary can be made by making two or three small loops in one or the other of the potential leads - that is, one or the other of the conductors $t$ and $u$-and placing them closer together or farther apart, as occasion may demand. These loops must be so placed that the 
mutual inductance between them and the conductor carrying the main part of the test current is negligibly small. In the figure the potential leads are shown slightly separated for the sake of clearness. In use they are twisted together to eliminate as far as possible the effects of mutual inductance between them and other parts of the system. The adjustments of the bridge may be carried out in different ways, though in general it is better to use direct current and make resistance adjustments as outlined on page 585 before making any attempt at adjusting the inductances. Then, with alternating current, inductance adjustments may be carried out as follows: (a) With the normal connections adjust $M$ so that the galvanometer indicates a zero current; (b) with the currents supplied through leads connected to $n$ and $n^{\prime}$ adjust the inductances in $s$ or $v$, to give a zero current in the galvanometer; (c) with the current supplied through the regular current leads and with the connector $Z$ removed, adjust the inductances in $t$ or $u$ so as to give a zero current through the galvanometer; (d) with the normal connections, again adjust $M$ so as to give a zero current in the galvanometer. If this requires much change in $M$, the series of adjustments should be repeated, using those already made as first approximations.

It will be observed that except for a preliminary adjustment, the method of making the inductance adjustments is the same as that given above for making the resistance adjustments.

If the resistances $X$ and $Y$ change appreciably on changing from direct to alternating current, a balance can not be established without a further adjustment of the resistances. This adjustment can be made by resetting the main and auxiliary resistance ratios and the change required is the same as the change in the ratio of $X$ to $Y$ on changing from direct to alternating current of the frequency used.

Where alternating current only is used in making the adjustments, we obtain the ratio of the resistances of the four-terminal conductors at only one frequency, unless, of course, a second adjustment is made with a different frequency. Also, unless some special device is used we can not tell, except by trial, what changes should be made. Consequently, more time is required for making 
the comparisons than where we begin by making the resistance adjustments using direct current. Here we have assumed the use of a vibration galvanometer. At the usual commercial frequencies other detectors are lacking in sensitivity or have other defects which make them unsuitable for use in this work. Even the vibration galvanometer used must have certain characteristics not common to all instruments of that type. First, the moving system must be practically nonmagnetic; otherwise it will be set in vibration by the "stray" magnetic field, which necessarily has the frequency to which the moving system is tuned. Second, the vibration galvanometer must be so designed and constructed as to have a high voltage sensitivity with an external resistance equal to the resistance of the bridge between the points $o$ and $o^{\prime}$. This matter requires care, since, on account of the back emf, ${ }^{28}$ we can not divide the current sensitivity by the resistance of the galvanometer plus the resistance of the bridge and assume that this gives the voltage sensitivity under the conditions of use.

In bridge measurements, with direct current, substitution methods have certain well-known advantages and are in common use where a number of standards of the same denomination are to be compared. When alternating current is used the advantages of substitution methods are much more pronounced. This is true whether the resistances are high, in which case capacities between the defferent parts of the system including the room in which the apparatus is located must be considered, or whether the resistances are low, in which case the mutual inductance between that part of the system which carries the large current and all other parts becomes a matter of real importance. Since, in addition to other advantages, the substitution method eliminates the effect of certain of the mutual inductances, it should be used, in most cases at any rate, where the standards to be compared are of the same denomination. A general discussion of the substitution method, or of the establishment and use of known ratios in measurements with the Thomson bridge or of devices which may be employed to simplify the calculations are matters which can not be considered in this paper. 


\section{SUMMARY}

I. The conditions which must necessarily be fulfilled in order that the resistance of a four-terminal conductor be definite are pointed out.

2. The additional conditions which must be fulfilled in order that the inductances as well as the resistances be definite are pointed out.

3. The theorem regarding the reciprocal relation obtained on interchanging the current and potential connections is discussed and applied in showing the relations between the resistances of a four-terminal conductor. It is also applied in showing the relations between the resistances and the relations between the inductances in the Thomson bridge.

4. It is shown that on using the four terminals in different combinations, three and only three values for the resistance are obtained, and that one of these is the sum of the other two.

5. Several devices which may be employed to increase the definiteness of the resistances are discussed, and it is pointed out that a symmetrical arrangement of the current and potential connectors together with the use of branched potential connectors makes the resistance sufficiently definite for the most precise measurements, even in the case of conductors which are to carry very large currents.

6. Some of the ideas which are discussed regarding resistances and inductances are embodied in the design of a resistance standard to carry a fairly large alternating current.

7. The theory of the Thomson bridge using linear four-terminal conductors is given and some of the different ways of determining or eliminating the correction terms are discussed.

8. The way in which the adjustments are carried out in the precision resistance comparisons at the Bureau of Standards is described.

9. Expressions are derived for the sensitivity of the combination of 'Thomson bridge and D'Arsonval galvanometer, with the motion of the coil critically damped. 
ro. The theory of the Thomson bridge where the four-terminal conductors are not linear is discussed, and it is shown that with the adjustments which are regularly made the same simple relations exist between the resistances whether the four-terminal conductors are linear or nonlinear.

II. Where alternating current is used it is shown that if certain adjustments are made the bridge can be balanced, and if we can neglect certain of the mutual inductances we have definite relations between the resistances and inductances of the main ratio coils and the four-terminal conductors, and the frequency. Where the time constants of all four of these conductors are small it is shown that the relation between the resistances and inductances are practically independent of the frequency. In this case if the bridge is balanced first with direct current and then with alternating current, the change in the ratio of the resistances of the fourterminal conductors on changing from direct to alternating current is obtained.

WASHINGTON, March 8, I9I2. 